\title{
The perception of pure and mistuned musical fifths and major thirds: Thresholds for discrimination, beats, and identification
}

\author{
Joos Vos \\ Institute for Perception TNO, Soesterberg, The Netherlands
}

\begin{abstract}
In Experiment 1, the discriminability of pure and mistuned musical intervals consisting of simultaneously presented complex tones was investigated. Because of the interference of nearby harmonics, two features of beats were varied independently: (1) beat frequency, and (2) the depth of the level variation. Discrimination thresholds (DTs) were expressed as differences in level $(\Delta \mathrm{L})$ between the two tones. DTs were determined for musical fifths and major thirds, at tone durations of 250,500 , and $1,000 \mathrm{msec}$, and for beat frequencies within a range of .5 to $32 \mathrm{~Hz}$. The results showed that DTs were higher (smaller values of $\Delta L$ ) for major thirds than for fifths, were highest for the lowest beat frequencies, and decreased with increasing tone duration. Interaction of tone duration and beat frequency showed that DTs were higher for short tones than for sustained tones only when the mistuning was not too large. It was concluded that, at higher beat frequencies, DTs could be based more on the perception of interval width than on the perception of beats or roughness. Experiments 2 and 3 were designed to ascertain to what extent this was true. In Experiment 2, beat thresholds (BTs) for a large number of different beat frequencies were determined. In Experiment 3, DTs, BTs, and thresholds for the identification of the direction of mistuning (ITs) were determined. For mistuned fifths and major thirds, sensitivity to beats was about the same. ITs for fifths and major thirds were not significantly different; deviations from perfect at threshold ranged from about 20 to 30 cents. Comparison of the different thresholds revealed that DTs are mainly determined by sensitivity to beats. Detailed analysis, however, indicated that perception of interval width is a relevant aspect in discrimination, especially for the fifths.
\end{abstract}

One of the classic problems of music theory is that of how to tune our 12-note chromatic scale. This tuning problem takes a very concrete form in the fixed-pitch keyboard instruments such as the organ, the harpsichord, and the piano. Since perfect fifths and pure major thirds are incompatible within one tuning system, it is inevitable that one introduces temperaments in which the fifths and/or major thirds are adjusted, that is, mistuned to some extent.

For complex tones, mistuned intervals are characterized by small frequency differences between those harmonics which coincide completely in pure intervals. [In this paper, interval represents a harmonic interval, in which a low tone (tone 1) and a high tone (tone 2) are presented simultaneously.] The interference of these just-noncoinciding harmonics gives rise to the perception of beats or roughness (see Helmholtz, $1877 / 1954$, chap. 10). When the interfering harmonics have equal amplitude, these beats are most prom-

This research was supported by Orant 15-29-05 from the Netherlands Organization for the Advancement of Pure Research (Z.W.O.). The author is indebted to Rudolf Rasch and to Reinier Plomp, who made critical comments on earlier versions of this paper. Thanks are also due Ben van Vianen, for his participation in running the third Experiment. Requests for reprints should be sent to Joos Vos, Institute for Perception TNO, Kampweg S, 3769 DE Soesterberg, The Netherlands. inent. In line with current models on tonal or sensory consonance, that is, the perception of consonance for isolated intervals (Kameoka \& Kuriyagawa, 1969; Plomp \& Levelt, 1965; Terhardt, 1976), we propose that the subjectively experienced out-oftuneness in music may in some way be related to the perception of beats. Since it is one aspect in the complex process of evaluating different tuning systems, it may be worthwhile to know our sensitivity to mistuning.

It was the aim of the present study to investigate the perception of pure and mistuned fifths and major thirds, particularly with respect to (1) discriminability between pure and mistuned intervals, (2) sensitivity to beats, and (3) identification of the direction of mistuning, that is, whether the interval will have been stretched or compressed. Before we give a detailed description of the experimental method, we shall elaborate the musical, physical, and psychophysical aspects that are relevant to our experimental study.

\section{MUSIC THEORY}

In Western music, consonant intervals such as the octave, fifth, and major third play an important role. A consonant interval is a musical interval in which 
the ratio of the fundamental frequencies of tones 1 and 2 can be described by means of small integers: $1: 2$ for the octave, $2: 3$ for the fifth, and $4: 5$ for the major third. In music theory, a major third equals the ratio between four fifths and two octaves:

$$
\beta=\alpha^{4} / \omega^{2},
$$

in which $\alpha, \beta$, and $\omega$ are the ratios of the fundamental frequencies of the fifth, major third, and octave, respectively. In Equation 1, we have two degrees of freedom, but given that the perfect octave $(\omega=2 / 1)$ is an axiom in all tuning systems, Equation 1 can be reduced to:

$$
\beta=\alpha^{4} / 4 .
$$

If the fifth is perfect $(\alpha=3 / 2)$, the major third is a Pythagorean major third (81/64), one syntonic comma $(81 / 80)$ larger than the pure major third. If the major third is pure $(\beta=5 / 4)$, the fifth equals $5^{1 / 4}$, or 1.495349 . This illustrates that it is not possible to construct tuning systems in which both the fifths and major thirds are pure. Either the fifths or the major thirds, or both, have to be mistuned. A mistuned major third, $\beta$, comprises the pure major third and a very small tempering interval, $b(\beta=5 b / 4)$. Likewise, a mistuned fifth, $\alpha$, comprises the pure fifth and a small interval a $(\alpha=3 a / 2)$. Substituting $(5 b / 4)$ and $(3 a / 2)$ in Equation 2 for $\beta$ and $\alpha$, respectively, results in:

$$
5 b / 4=(3 a / 2)^{4} / 4,
$$

from which it follows that

$$
b=(81 / 80) a^{4} .
$$

Both in musical and perceptual contexts, it is considered to be more appropriate to convert frequency ratios of musical intervals into logarithmic quantities (Barbour, 1940; Pikler, 1966; Young, 1939). Logarithmic conversion of Equation 4 results in

$$
B=S+4 A,
$$

in which $\mathrm{S}$ equals the syntonic comma. The relation between the mistuning of the major third and the mistuning of the fifth, as given in Equation 5, is depicted in Figure 1. Mistuning is expressed both with $S$ and with the cent as unity. The cent is defined as the interval between two tones having as a basic frequency ratio the 1,200 th root of 2 . Figure 1 clearly illustrates that there is only a restricted range in which an increase in mistuning of the major third results in a decrease in mistuning of the fifth, and vice versa. Only this range, which is printed boldly

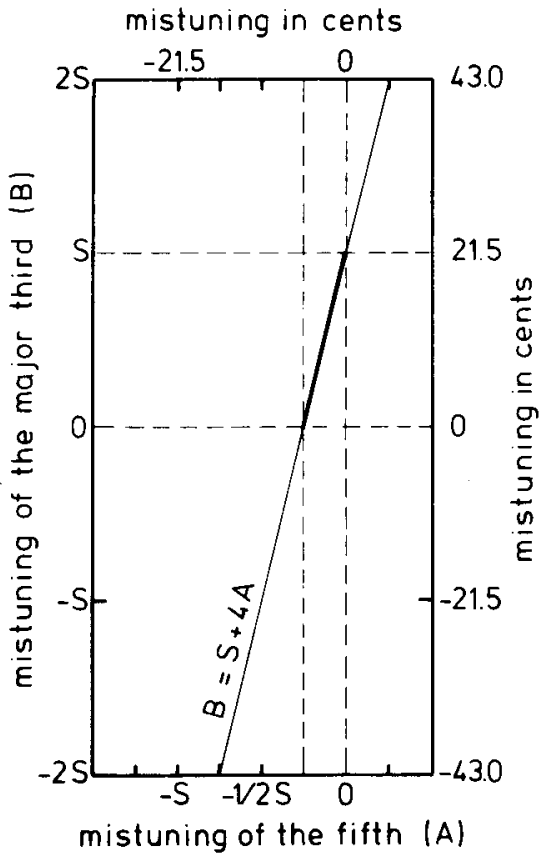

Figure 1. Relation between mistuning of the major third and mistuning of the fifth. Mistuning is expressed both with the syntonic comma (S) and with the cent as unity. The range in which the purity of both the fifths and the major thirds can be optimized is in bold print.

in Figure 1, is relevant for optimizing the purity of both the fifths and the major thirds.

\section{Physical Aspects}

\section{Beat Frequency}

In a consonant interval, in which the ratio of the fundamental frequency $f_{1}$ of tone 1 and the fundamental frequency $f_{2}$ of tone 2 is equal to $p: q$, with $p$ and $q(p<q)$ small integers, the nq ${ }^{\text {th }}$ harmonic of tone 1 coincides with the npth harmonic of tone 2 $(\mathrm{n}=1,2, \ldots)$.

In mistuned intervals, however, where $f_{1}: f_{2}$ slightly departs from $p: q$, these harmonics do not coincide exactly in frequency. The superposition of the different pairs of just-noncoinciding harmonics with frequencies of $\mathrm{nqf}_{1}$ and $\mathrm{npf}_{2} \mathrm{~Hz}$ and equal amplitudes is equal to

$$
\begin{aligned}
& \sin 2 \pi\left(n q f_{1}\right) t+\sin 2 \pi\left(n p f_{2}\right) t \\
& \quad=2 \cos \left[2 \pi^{1 / 2}\left(n \operatorname{nf}_{2}-n q f_{1}\right) t\right] \cdot \sin \left[2 \pi^{1 / 2}\left(n \operatorname{nf}_{2}+n q f_{1}\right) t\right] .
\end{aligned}
$$

For a given value of $n$, the compound waveform can be interpreted as a tone with frequency $1 / 2\left(\mathrm{npf}_{2}+\right.$ nqf $\left._{1}\right) \mathrm{Hz}$, which is the mean of the frequencies of the two harmonics, and a slowly fluctuating amplitude 


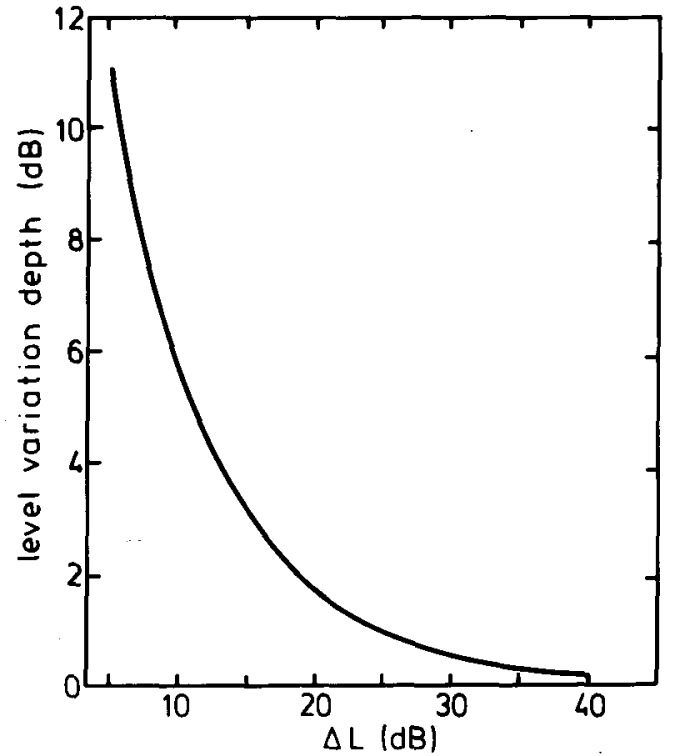

Figure 2. Depth of level variation of two beating tones, given as a function of the level difference $\Delta L$ of tones 1 and 2 . $\Delta L$ equals $0 \mathrm{~dB}$ when the spectral envelopes of tones 1 and 2 coincide.

given by $\mid 2 \cos 2 \pi \frac{1}{2}\left(\right.$ npf $\left._{2}-n q f_{1}\right) t \mid$. Since this amplitude varies $\left(\mathrm{npf}_{2}-\mathrm{nqf}_{1}\right)$ times per second between 0 and 2 , the frequency of the beating sensation $f_{b n}$ for the $n^{\text {th }}$ pair of interfering harmonics is equal to

$$
f_{b n}=\left|n p f_{2}-n q f_{1}\right| \text {. }
$$

If npf $_{2}-$ nqf $_{1}>0$, the musical interval is stretched $\left(f_{2} / f_{1}>q / p\right)$, if npf $_{2}-$ nqf $_{1}<0$, the musical interval is compressed $\left(f_{2} / f_{1}<q / p\right)$. In our experiments, the degree of mistuning of the intervals will be expressed as the beat frequency for the lowest pair of justnoncoinciding harmonics $(n=1)$. The relationship between this beat frequency $f_{b}$ and deviation $T$ (in cents of the mistuned interval from a perfect interval is given by

$$
f_{b}=\left|q f_{1}\left(2^{T / 1,200}-1\right)\right|,
$$

or simply by

$$
f_{b}=\left|q f_{1} T / 1,731\right| \text {. }
$$

Equation 8 shows that the beat frequency depends on the amount of mistuning $T$, the value of $q$, and the frequency of the fundamentals. From Equation 8 , the upper boundary of those beat frequencies which are significant in music perception research can be computed. For example, the beat frequency of a major third with fundamental $f_{1}$ of $349 \mathrm{~Hz}$ (musical F4), which is tuned 21.5 cents sharp, equals $21.8 \mathrm{~Hz}$.

\section{Level Variation Depth}

The amplitude $p(t)$ of the two interfering harmonics with waveforms $a_{1} \sin 2 \pi q f_{1} t$ and $a_{2} \sin 2 \pi p f_{2} t$ equals:

$$
p(t)=\left(a_{1}^{2}+a_{2}^{2}+2 a_{1} a_{2} \cos 2 \pi f_{b} t\right)^{1 / 2} .
$$

The maximum and minimum values of $p(t)$ are $\left(a_{1}+a_{2}\right)$ and $\left(a_{1}-a_{2}\right)$, respectively. The level variation depth in decibels of the beating harmonics is given by

$$
D=20 \log \left|\frac{a_{1}+a_{2}}{a_{1}-a_{2}}\right|=20 \log \left[\frac{10^{\Delta L} / 20+1}{10^{A L} / 20-1}\right],
$$

in which $\Delta \mathrm{L}$ is the absolute level difference between $a_{1}$ and $a_{2}$ in decibels $\left[\Delta L=\left|20 \log \left(a_{1} / a_{2}\right)\right|\right] . D$ approaches $\infty$ for $a_{2} \rightarrow a_{1}$. In Figure 2, the relation between the level difference $\Delta \mathrm{L}$ and level variation depth $\mathrm{D}$, as defined in Equation 10, is given.

\section{Psychophysical Aspects}

\section{The Perception of Beats}

To our knowledge, no experimental results on thresholds for the perception of beats for intervals consisting of two complex tones have been reported. Only the discrimination of beats, caused by adding two sinusoids of nearly the same frequency, has been studied.

As a function of beat rate, Riesz (1928) found a Ushaped curve for the detection threshold of intensity fluctuations, with a minimum for about $3 \mathrm{~Hz}$. Further experiments at this beat frequency revealed that sensitivity increases with increasing level above absolute hearing threshold (sensation level) up to $60 \mathrm{~dB}$, and remains constant at higher levels. This effect was verified by Harris (1963). In addition, Harris found that the threshold varies in the same way as a function of sensation level both for beats resulting from two interfering sinusoids and for sinewave amplitude modulation (AM) of one sinusoid.

Since sensitivity for the perception of beats is high, that is, the difference between $a_{1}$ and $a_{2}$ at threshold is large, the amplitude $p(t)$ as given in Equation 9 can be approximated for threshold conditions by:

$$
p(t)=a_{1}+a_{2} \cos 2 \pi f_{b} t . \quad\left(a_{1}>a_{2}\right)
$$

Since this function is identical to the amplitude variation of an AM tone, we can relate the results from studies on AM tones to those of Harris (1963) and Riesz (1928). As for two tones, the level variation threshold for $\mathbf{A M}$ tones decreases with increasing modulation frequencies up to $4 \mathrm{~Hz}$ and increases again for modulation frequencies above this value (Ebel, 1952; Terhardt, 1974; Zwicker, 1952). 
Maiwald (1967) and Schöne (1979) have shown that the level variation threshold decreases with increasing sound pressure level (SPL). Zwicker (1952) found that this effect does not depend on modulation frequency.

\section{The Perception of Interval Width}

Musical interval recognition. Using simultaneous complex tones, Plomp, Wagenaar, and Mimpen (1973) showed that trained listeners are able to categorize the 12 musical intervals between $\mathrm{C} 4$ and $\mathrm{C5}$, if tone duration is $120 \mathrm{msec}$, with a mean accuracy of $83 \%$. In a similar study, Killam, Lorton, and Schubert (1975) found that their subjects reached a mean score of $67 \%$ correct. The sharpness of transitions between the categories has been successfully determined by means of successive sine-wave tones. In these experiments, subjects were presented with ascending intervals, their widths being varied independently in small steps of 12.5 to 20 cents. A number of studies have demonstrated that, also in such conditions, trained listeners are able to use musical-interval categories consistently, and that their transitions between adjacent categories are sharp and well defined (Burns \& Ward, 1978; Siegel \& Siegel, 1977a, 1977b).

Identification of the direction of mistuning. Identification of the direction of mistuning was investigated extensively by Stumpf and Meyer (1898). To make their results more accessible, we reanalyzed the data by transforming the frequency differences between the tones into deviations (in cents) of the mistuned intervals from the pure intervals, and by transforming the correct response percentages for the stretched and compressed conditions into $\mathrm{z}$ scores. From these values, thresholds were determined by means of linear regression functions. For melodic major thirds, fifths, and octaves, averaged over sine-wave tones and complex tones, thresholds were 3,5 , and 4 cents, respectively. In the same way, the data from the harmonic intervals were reanalyzed. The results are shown in Figure 3 for the major third, fifth, and octave, separately. The amount of mistuning, which resulted in $75 \%$ correct compressed and $75 \%$ correct stretched responses, is indicated by arrows. In these conditions, thresholds for the major third and octave are 12 and 20 cents, respectively, and thus considerably higher than in the melodic conditions. This had also been observed by Delezenne (1827). It should be noted, however, that, in Stumpf and Meyer (1898), determination of thresholds for melodic and harmonic intervals was confounded with stimulus complexity. In the melodic conditions, the frequency of either the low or the high tone was fixed, whereas in the harmonic conditions, both tones could be varied in frequency. The low threshold for the fifth $(=4$ cents, see Figure $3 b$ ) can also be attributed to stimulus simplicity since in this particular harmonic con-
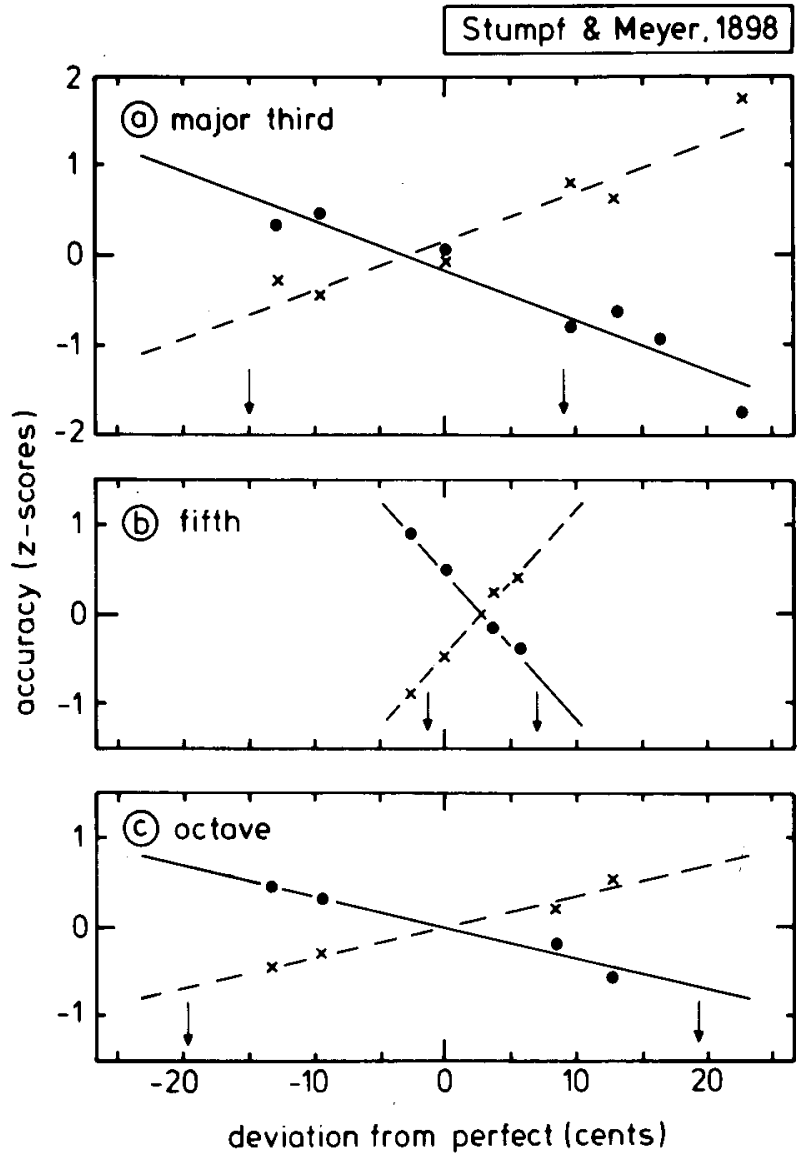

Figure 3. Reanalysis of datn from Stumpf and Meyer (1898), who investigated identification of the direction of mistuning for several musical intervals. Frequency differences between the tones are transformed into deviations (in cents) of the mistuned intervals from the perfect intervals. Percentage correct responses for the stretched $(x)$ and compressed $(\bullet)$ conditions are converted to $z$ scores. Thresholds (75\% correct), computed by means of linear regression functions, are indicated by arrows. For harmonic intervals, results are presented for the major third, fifth, and octave in panels $\mathbf{a}, \mathbf{b}$, and $\mathrm{c}$, respectively.

dition only the high tone was varied. The effect of stimulus complexity has recently been demonstrated by Zatorre and Halpern (1979), who found for their subjects that consistency of labeling data was entirely dependent on the constancy of the lower tone.

The idea that thresholds for the perception of interval width are more likely to range between 10 and 20 cents, or even between 10 and 30 cents, than between 4 and 10 cents is in line with adjustment thresholds for musical intervals consisting of simultaneous sine-wave tones (Moran \& Pratt, 1926), and with adjustment thresholds for musical intervals consisting of successive sine-wave tones (Rakowski, Note 1).

\section{The Rationale Behind the Experiments}

In Experiment 1, discrimination between pure and mistuned fifths and pure and mistuned major thirds was investigated. Two features of the beats were 
varied: (1) beat frequency, representing the amount of mistuning (see Equations 7 and 8), and (2) level variation depth of the beats (see Equation 10). We shall learn that, for high beat frequencies, thresholds at which subjects correctly discriminated between pure and mistuned complex-tone intervals $75 \%$ of the time deviated from the well-known U-shaped curve for the detection threshold of beats. Thresholds for beats in complex-tone intervals, however, do not necessarily have to be similar to those caused by superposition of two sinusoids of nearly the same frequency. In Experiment 2, we therefore determined thresholds for beats in complex-tone intervals for a large number of beat frequencies, including those of the various mistuned intervals from Experiment 1.

The main results from the second experiment were (1) that sensitivity to beats in complex tone intervals does not have a clear maximum such as the one found with superimposed sinusoids, and (2) that there was at least one condition in which the pattern of the beat threshold strongly deviated from the pattern of the discrimination threshold. The latter finding supported our notion that, apart from beats, the perception of differences between the size of the mistuned interval and that of the pure interval may have contributed to the discrimination thresholds.

Experiment 3 was designed to obtain more direct evidence that both the perception of beats and the perception of interval width were relevant aspects in discrimination. In this experiment, not only discrimination and beat thresholds, but also thresholds for identification of the direction of mistuning were determined.

\section{EXPERIMENT 1}

It is needless to say that tone duration is a relevant parameter in music. Moreover, while listening to performed music, it can be easily noticed that the perceptibility of beats is enhanced at longer tone durations. Therefore, we decided to determine discrimination thresholds at various tone durations, ranging from 250 to $1,000 \mathrm{msec}$.

\section{Method}

\section{Stimuli}

The stimuli were intervals consisting of two complex tones, 1 and 2 , with fundamental frequencies of $f_{1}$ and $f_{2} \mathrm{~Hz}$, respectively. The frequency ratio $f_{1}: f_{2}$ was equal to or slightly different from $p: q$. We presented musical fifths $(p=2 ; q=3)$ and musical major thirds $(p=4 ; q=5)$. The complex tones 1 and 2 consisted of 20 harmonics with amplitudes according to:

$$
p(t)=\sum_{n=1}^{n=20} \frac{1}{n} \sin \left(2 \pi n f t+\phi_{n}\right)
$$

This resulted in a stimulus with a spectral envelope slope of $-6 \mathrm{~dB} /$ octave. The phase $\left(\phi_{n}\right)$ of the individual harmonics was chosen randomly. Because of this, the waveforms of tones 1 and
2, although having the same spectral envelope, were different. The overall level of tones 1 and 2 together was $85 \mathrm{~dB}$ SPL. This level was measured with the help of an artificial ear (Briel \& Kjaer, Type 4152). Rise and decay times of the tone bursts, defined as the time interval between $10 \%$ and $90 \%$ of the maximum amplitude, were 40 and $20 \mathrm{msec}$, respectively. Level variation depth of the beating harmonics was obtained by attenuation of tone 1 or tone 2 by $\Delta \mathrm{L} d B$. When the spectral envelopes of the two tones coincide, that is, when the amplitudes of the just-noncoinciding harmonics are equal, level variation depth is maximal. We defined $\Delta \mathrm{L}$ to be $0 \mathrm{~dB}$ for this condition. The spectral contents of our intervals in the conditions in which $\Delta L$ equals $0 \mathrm{~dB}$ are depicted in the left-hand panels of Figure 4 . In these panels, only the levels of the first 10 harmonics of tone 1 and the first 7 or 8 harmonics of tone 2 are given. The levels are plotted relative to the level of the first harmonic of tone 1 . Coincidence of spectral envelopes occurs when the level of tone 2 is $20 \log (q / p) d B$ lower than the level of tone 1. Increments of $\Delta L$ from the base conditions, as illustrated in the left-hand panels of Figure 4, always result in smaller level variation depths. In the middle panels of Figure 4, the spectral contents of our intervals are given for cases in which $\Delta L=15 \mathrm{~dB}$ and the level of tone $2\left(\mathrm{~L}_{2}\right)$ is lower than the level of tone $1\left(\mathrm{~L}_{1}\right)$. In the righthand panels, the levels of the harmonics are presented relative to the level of the first harmonic of tone 2 . In the latter conditions, when $L_{1}<L_{2}$, $\Delta L$ equals $15 \mathrm{~dB}$ again.

\section{Apparatus}

A block diagram of the apparatus is given in Figure 5. The experiments were run under the control of a PDP-11/10 computer. Tones 1 and 2 were generated in the following way. One period of the waveforms of tones 1 and 2 was stored in 256 discrete samples (with 10-bit accuracy) in external revolving memories. These recirculators could be read out by digital-to-analog converters. Sampling rates were determined by pulse trains, derived from frequency generators 1 and 2 . After gating, the tones were filtered by Krohn-Hite filters (Model 3341) with the function switch in the low-pass RC mode and with a cutoff frequency of $8 \mathrm{kHz}$. The sound-pressure levels of the tones were controlled by programmable attenuators. After appropriate attenuation, the tones were mixed and fed to two headphone amplifiers. The signals were presented diotically (same signal to both ears) by means of Beyer DT $48 \mathrm{~S}$ headphones. Subjects were seated in a soundproof room.

\section{Subjects}

Four musically trained subjects were tested over eight sessions, each session on a different day. Two were students from the Institute of Musicology at Utrecht, who were paid for their participation; the other two were the author and a colleague. Since the experiment aimed at studying a musical-perceptual competence, the choice of musically trained subjects was a deliberate one. Moreover, our subjects were experienced in performing auditory tasks, and instruction was facilitated by the possibility of using musical terminology. We decided to present our stimuli to a rather small number of subjects because, due to the nature of the experimental task, we did not expect to find large discrepancies between the thresholds of the different subjects.

\section{Experimental Design}

The independent variables were: (1) tone duration (250, 500, and 1,000 msec); (2) musical interval (fifth and major third); (3) beat frequency of the first pair of just-noncoinciding harmonics $(.5,1,2,4,8,16$, and $32 \mathrm{~Hz}$ ); (4) attenuation of tone 1 or tone 2 ; (5) direction of mistuning (stretched vs. compressed interval). Description of the discrimination threshold, being the dependent variable, is given below. For a tone duration of $250 \mathrm{msec}$ and beat frequencies of .5 and $1 \mathrm{~Hz}$, and for a tone duration of $500 \mathrm{msec}$ and a beat frequency of $.5 \mathrm{~Hz}$, no discrimination thresholds were determined because these stimuli contained less than half a beating period. This means that, with respect to tone duration, we have an 


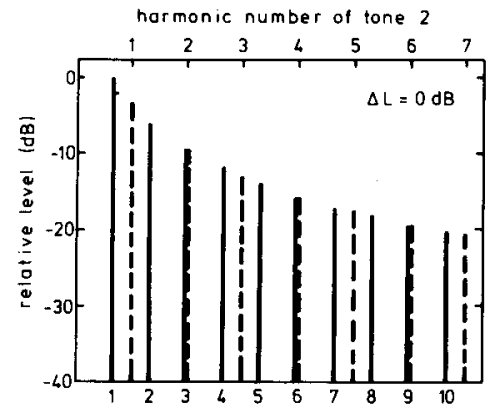

har monic number of tone 1

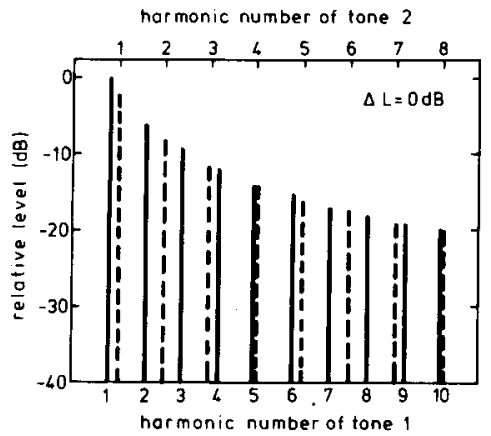

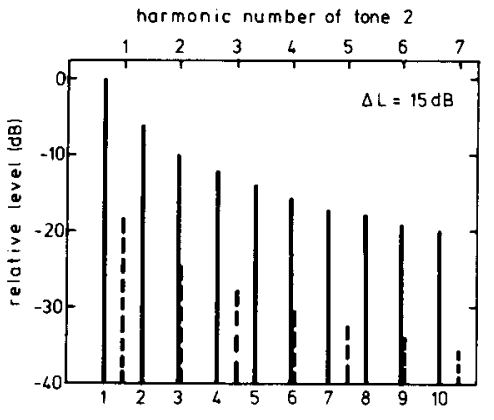

harmonic number of tone 1

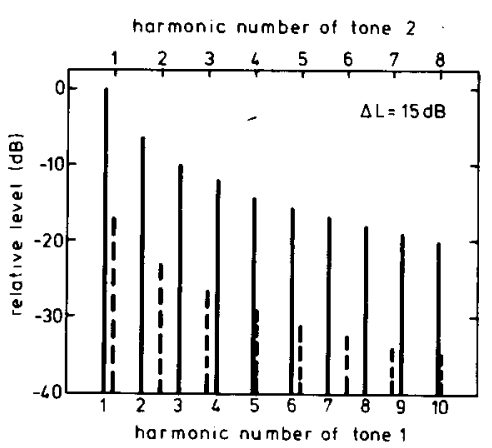

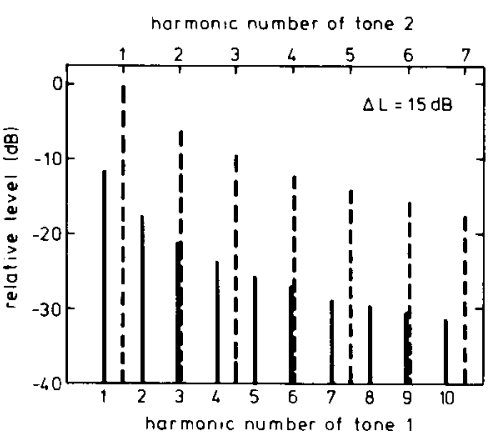

(a)

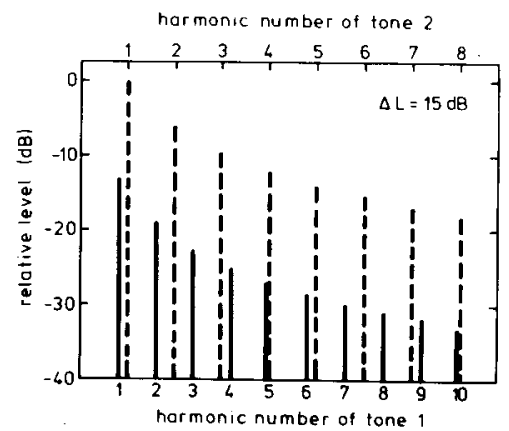

Figure 4. Presentation of the spectral contents of various fifths (panel a) and major thirds (panel b). The levels of the first 10 harmonics of tone 1 (bottom hoxizontal) and the first 7 or 8 harmonics of tone 2 (uppex horizontal) are given on a linear frequency scale. In the left-hand and middle panels, the levels of the harmonics are plotted relative to the level of the first harmonic of tone 1 . In the right-hand panels, the levels are given relative to the level of the first harmonic of tone 2.

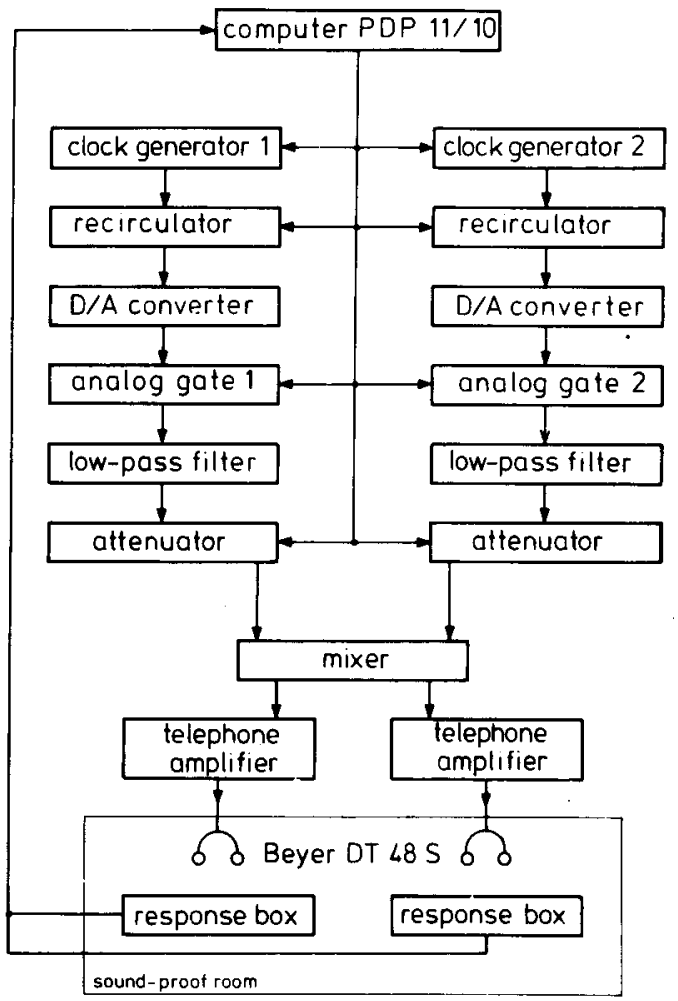

Figure 5. Block diagram of the apparatus used. incomplete factorial design. Analyses of variance (ANOVAs) on the interactions of tone duration with the other variables could be performed for the five beat rates from 2 to $32 \mathrm{~Hz}$.

\section{Data Acquisition and Data Reduction}

The task for the subject was to discriminate between pure and mistuned musical intervals in a 2-AFC paradigm. By means of the method of constant stimuli, all stimulus combinations were presented 10 times for six different values of $\Delta L$ in equal steps of 4 or $5 \mathrm{~dB}$. For every stimulus combination, the raw scores for different $\Delta L$ values were transformed to percentage correct. Since the response patterns of the stretched and compressed intervals were similar in almost all cases, the percentages of these two conditions were combined. On the assumption that the scores, for a given set of $\Delta L$ values, are cumulatively normally distributed (Kling \& Riggs, 1972, pp. 11-14), these percentages were converted to $z$ scores. The discrimination threshold, that is, the value of $\Delta \mathrm{L}$ at which a subject responded correctly in $75 \%$ of the cases, was obtained by solving the linear regression function $z=a+b \Delta L$ for $\Delta \mathrm{L}$ when $\mathrm{z}$ equals .67. Computation of coefficients of determination $\left(r^{2}\right)$ for all linear regression functions showed that the explained variability in $\mathrm{z}$ by $\Delta \mathrm{L}$ and the linear rule, was more than .75 in $73 \%$ of the cases. Moreover, $r^{2}$ was about the same for all conditions and all subjects. Mean $r^{2}$ was .80 .

\section{Procedure}

Every trial consisted of a comparison of a pure interval and a mistuned interval. The mistuned interval was presented first in half of the trials and second in the other half of the trials. Between the intervals was a silent period of $1.5 \mathrm{sec}$. By means of a response box, the subject indicated whether the first or the second interval was mistuned. Feedback was given by a red light above the correct button. The next trial was presented $1.5 \mathrm{sec}$ after the response to the preceding trial. Dependent upon stimulus duration, 
which was held constant within the series, a series comprised 240 , 288 , or 336 trials. The presentation order of the different series was randomized. Within a series, which lasted 15 to $25 \mathrm{~min}$, the stimuli were presented in a random order. At the beginning of a series, new waveforms of tones 1 and 2 , with different $\phi_{n}$ values, were determined. The fundamental frequencies were varied from trial to trial in such a way that the central frequency $f_{c}[=$ the geometric mean $\left.\left(f_{1} f_{2}\right)^{1 / 2}\right]$ of the pure interval was equal to $370 \mathrm{~Hz}$ $\pm 50, \pm 100, \pm 150$, or \pm 200 cents, in a random order. As a result, all fundamental frequencies fell within a range of $261 \mathrm{~Hz}$ (musical C4) and $523 \mathrm{~Hz}$ (musical C5). The $f_{1}$ of the pure interval equaled

$$
f_{1}=f_{c} /(q / p)^{1 / 2}
$$

In the mistuned interval, one of the fundamental frequencies was equal to the corresponding fundamental frequency in the pure interval, whereas the other one was altered. Within a series, the frequencies of tones 1 and 2 were altered equally often, the particular order being determined by sampling without replacement. For the mistuned interval, either a new $f_{1}$ was calculated by means of

$$
f_{1}(\text { mistuned })=\left(p \cdot f_{2} \pm f_{b}\right) / q,
$$

or a new $f_{2}$ was calculated by

$$
f_{2}(\text { mistuned })=\left(q \cdot f_{1} \pm f_{b}\right) / p .
$$

Within a series, the particular order of stretched vs. compressed intervals (signs in Equations 14 and 15) was randomized. A session comprised five or six series. During the first and second sessions, in which subjects were trained, the adequate range of $\Delta \mathrm{L}$ was determined. The choice of $\Delta \mathrm{L}$ demanded careful planning because the series of six equally spaced values of $\Delta \mathrm{L}$ had to cover the subjects' transition range between chance performance and $100 \%$ correct responses. If the presented range of $\Delta \mathrm{L}$ values did not result in scores around $75 \%$, a better range was chosen. The range appeared to depend on the particular musical interval and on whether tone 1 or tone 2 was attenuated. In addition, the adequate range differed from subject to subject to a small extent. In most cases, the range of $\Delta \mathrm{L}$ was from 10 to $40 \mathrm{~dB}$. In the $\Delta \mathrm{L}<10 \mathrm{~dB}$ conditions, $L_{1}$ and $L_{2}$ were attenuated to a small extent to satisfy our criterion that the overall level of the interval should remain at a constant level of $85 \mathrm{~dB}$ SPL. The correction (C) in decibels of $L_{1}$ and $L_{2}$, being dependent on $\Delta L$ and on the musical interval $(q / p)$, is given by

$$
C=10 \log \left\{1+10^{[-\Delta L \pm 20 \log (q / p)) / 10}\right\} .
$$

The positive and negative signs apply to the $\mathrm{L}_{1}<\mathrm{L}_{2}$ and $\mathrm{L}_{1}>\mathrm{L}_{2}$ conditions, respectively.

\section{Results}

Discrimination thresholds (DTs) are presented in terms of level difference, $\Delta \mathrm{L}$, rather than of level variation depth, D (see Equation 10). This way of presentation was preferred because the exact contribution of the various pairs of beating harmonics to DT is not known a priori. Moreover, other sources such as the perception of waveform periodicity and combination tones may have influenced DT as well (Plomp, 1976).

DTs were subjected to an ANOVA [ 4 (subjects) $\times 3$ (tone duration) $\times 2$ (fifth or major third) $\times 2$ (attenua-

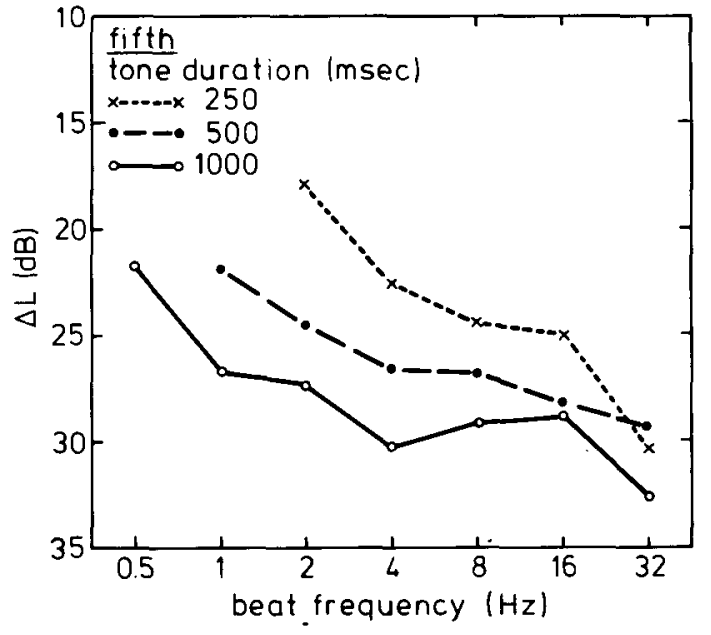

Figure 6. Thresholds for the discrimination between pure and mistuned fifths, plotted as a function of beat frequency, with tone duration as parameter.

tion of tone 1 or tone 2 ) $\times 5$ (beat frequencies of 2,4 , 8,16 , and $32 \mathrm{~Hz}$ ), all repeated measures]. Although individual differences occurred $[F(3,24)=94.0$, $\mathrm{p}<.00001$ ], a number of experimental variables affected our subjects' DTs in similar ways.

In general, mean threshold increases when tone duration becomes shorter $[\mathrm{F}(2,6)=68.4, \mathrm{p}<.0005]$. Tone duration is most effective at low beat frequencies, whereas the differences become less apparent at the highest beat frequency $[F(8,24)=12.3$, $\mathrm{p}<.00001]$. This interaction effect, being slightly dependent on musical interval $[F(8,24)=2.33$, $p<$ $.05]$, is given separately for the fifth and the major third in Figures 6 and 7, respectively. In these figures, DTs are plotted as a function of beat frequency with tone duration as parameter. The DTs for the fifths are lower than for the major thirds $[F(1,3)=$

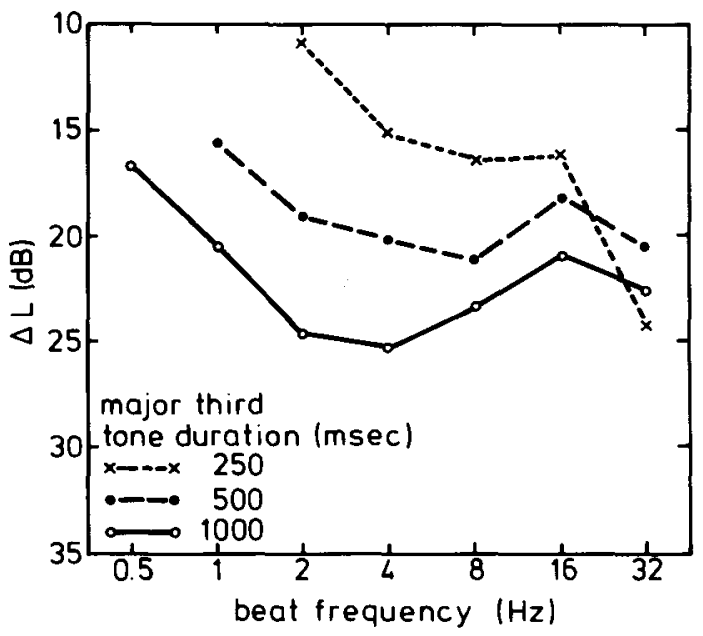

Figure 7. Thresholds for the discrimination between pure and mistuned major thirds, plotted as a function of beat frequency, with tone duration as parameter. 


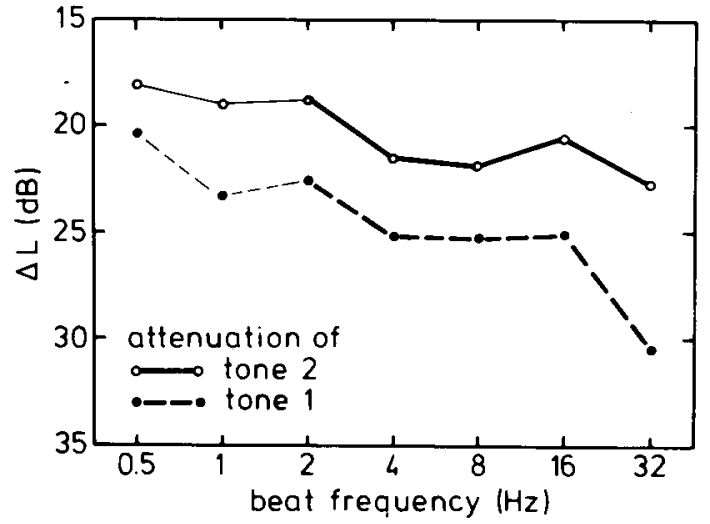

Figure 8. Discrimination thresholds, given as a function of beat frequency with attenuation of elther tone 1 or tone 2 as parameter. Mean values, connected by boldly printed lines, are based on thresholds from three different tone durations.

48.0, $\mathrm{p}<.005]$. Attenuation of tone 1 results in lower DTs than attenuation of tone $2[F(1,3)=63.2$, $p<.005]$. This effect depended to some degree on beat frequency $[F(4,12)=3.22, p<.05]$. In Figure 8 , where the mean DTs are plotted as a function of beat frequency with attenuation of either tone 1 or tone 2 as parameter, it is shown that this attenuation effect is due to the increased difference of the DTs at a beat frequency of $32 \mathrm{~Hz}$.

For the fifths, the effect of beat frequency is similar for all tone durations. The general trend is that these DTs decrease with increasing beat frequency, although three Newman-Keuls paired comparison tests (Winer, 1970), one for each tone duration, showed that DTs for beat frequencies of 4,8 , and $16 \mathrm{~Hz}$ are not significantly different.

For the major thirds, however, the effect of beat frequency is different (see Figure 7). At a tone duration of $1,000 \mathrm{msec}$, tests for trend (Winer, 1970) revealed that most of the variation caused by beat frequency is accounted for by the quadratic trend. The Newman-Keuls paired comparison test showed that the DTs for beat frequencies of $.5,1$, and $16 \mathrm{~Hz}$ are all significantly different from the DT at $4 \mathrm{~Hz}(\alpha=$ .05). A similar paired comparison test between the DTs for a tone duration of $500 \mathrm{msec}$ indicated that the mean threshold at $16 \mathrm{~Hz}$ is not significantly higher than it is at $8 \mathrm{~Hz}$.

\section{Discussion}

The ANOVA on the DTs for beat frequencies from 2 to $32 \mathrm{~Hz}$, as well as three ANOVAs, carried out on the DTs for each tone duration separately, showed that the main effects of musical interval, attenuation of tone 1 or tone 2 , and beat frequency were significant in all subgroups of data. In addition, tone duration and its interaction with beat frequency proved to be very effective. These effects will be discussed in detail.

\section{Musical Interval}

Mean thresholds for the detection of mistuned fifths were about $7 \mathrm{~dB}$ lower than they were for the detection of mistuned major thirds. On the assumption that the perception of various beating pairs of just-noncoinciding harmonics provides one of the most important cues in the discrimination task, we can explain at least part of this difference by refering to the fact that the mean level of interfering harmonics for the fifths was about $5 \mathrm{~dB}$ higher than it was for the major thirds. With the fifths, for example, when $L_{1}>L_{2}$, the level of the unattenuated third harmonic of tone 1 is $9.5 \mathrm{~dB}$ lower than the level of the fundamental (see middle panel of Figure $4 a$ ). When $L_{1}<L_{2}$, the level of the unattenuated second harmonic of tone 2 is $6.0 \mathrm{~dB}$ lower than the level of the fundamental (see right-hand panel of Figure 4a). For the major thirds, however, the corresponding values are substantially lower: 14 and $12 \mathrm{~dB}$, respectively. The mean difference of $5.25 \mathrm{~dB}$ in the levels of the interfering harmonics holds for all other pairs of just-noncoinciding harmonics. Our hypothesis that the DT depends on the SPL of the interfering harmonics is based on data presented by Maiwald (1967) and by Schöne (1979), who showed that the modulation threshold of AM sinusoids decreases with increasing SPL of the tones. Moreover, Zwicker (1952) found that this threshold decrease does not depend on modulation frequency. The combined results from Maiwald, Schöne, and Zwicker indicate that when the signal level increases from $\mathbf{4 0}$ to $80 \mathrm{~dB}$, the level variation depth D (Equation 10) at threshold decreases from about .85 to $.35 \mathrm{~dB}$. In terms of $\Delta \mathrm{L}$, this threshold decrease is $8 \mathrm{~dB}$. At low sensation levels within a range of 5 to $40 \mathrm{~dB}$, a similar relation between sensation level and threshold was found for beats resulting from the superposition of two sinusoids with a frequency difference of $3 \mathrm{~Hz}$ (Riesz, 1928). Since the literature cited above suggests that the effect of sensation level on the DT is rather small, it is unlikely that the difference between the DTs of the fifths and the major thirds can be completely explained by the mean level difference of $5.25 \mathrm{~dB}$ between the interfering harmonics.

There is a second factor, which may have also influenced the DTs for the fifths and the major thirds differently. For any beat frequency, $f_{b}$, the interval size of the mistuned fifths has to be changed to a greater extent than the interval size of the major thirds (see also Equation 8). From this equation it follows that this difference increases with beat frequency, being 28 cents when $f_{b}$ equals $32 \mathrm{~Hz}$. The relevance of this second factor is apparent in the trend for the differences between the DTs for the fifths and the major thirds to increase at the higher 
beat frequencies. The second factor will be discussed in detail in the section on the effect of beat frequency.

\section{Attenuation of Tone 1 or Tone 2}

That DTs depended on whether tone 1 or tone 2 was attenuated can be partly explained again by the fact that beats are most easily observed at higher levels of the interfering harmonics, as discussed above: the mean SPLs of the interfering harmonics in the $\mathrm{L}_{1}<\mathrm{L}_{2}$ conditions are $2.75 \mathrm{~dB}$ higher than in the $\mathrm{L}_{1}>\mathrm{L}_{2}$ conditions. Masking also seems to be a candidate for explaining part of the differences. It is a well-established fact that the masked threshold decreases as a function of the frequency separation of the masker and the maskee (see Plomp, 1976). In the $\mathrm{L}_{1}<\mathrm{L}_{2}$ condition, the frequency separation of the harmonics of tone 2 is a factor $q / p$ wider than the frequency separation of the harmonics of tone 1 in the $\mathrm{L}>\mathrm{L}_{2}$ condition. Thus, less masking of the various pairs of interfering harmonics would be expected in the $\mathrm{L}_{1}<\mathrm{L}_{2}$ conditions. This is supported by our data, since the DTs were lower in the $\mathrm{L}_{1}<\mathrm{L}_{2}$ conditions.

\section{Beat Frequency}

At tone durations of 500 and $1,000 \mathrm{msec}$, the DTs for beat frequencies higher than about 4 or $8 \mathrm{~Hz}$ tended to decrease when the interval was a fifth and tended to increase when the interval was a major third. We think that these particular patterns can be explained by the combined effect of two different processes, which have opposite effects on DT for beat frequencies from about $4 \mathrm{~Hz}$ on. The first mechanism is related to the perception of beats. Recall that both for two superimposed sinusoids and for AM tones, sensitivity to beats increases with increasing beat frequency up to about $4 \mathrm{~Hz}$ and decreases again for beat frequencies above this value. It is reasonable to assume a similar sensitivity for the perception of beats for our complex tones.

The second mechanism is based on the perception of interval width and is thus related to musical interval recognition. From Equation 8, it can be inferred that, for a constant beat frequency, the deviation of the mistuned intervals from the pure intervals, $|\mathrm{T}|$, decreases with increasing values of $f_{1}$. In Figure 9, the DTs for the different beat frequencies, as given in Figures 6 and 7, are replotted as a function of the corresponding values of mean $|\mathrm{T}|$, with tone duration as parameter. For beat frequencies of $32 \mathrm{~Hz}$, for example, the corresponding mean $|\mathrm{T}|$ of the mistuned fifths and the mistuned major thirds equaled 61 and 34 cents, respectively. We suppose that our subjects, especially in these conditions, identified the mistuned intervals, in at least a number of trials, as not belonging to the pure fifth or pure major third category. Thresholds for identification of the direction of mistuning (Stumpf \& Meyer, 1898) and adjustment thresholds for musical intervals (Moran \& Pratt, 1926; Rakowski, Note 1) suggest that this proposition is correct.

\section{Tone Duration}

There is a downward shift of the DTs with increasing tone duration. This shift is most pronounced for lower beat frequencies. For beat frequencies within a range of .5 to $16 \mathrm{~Hz}$, halving of tone duration of the fifth is equivalent to about quadrupling of beat frequency. For the major third, a simple rule cannot be given because of the strong quadratic trend in the data.

In our experiments, all subjects could discriminate between pure and mistuned intervals, if the mistuned intervals contained at least one half beating period. It

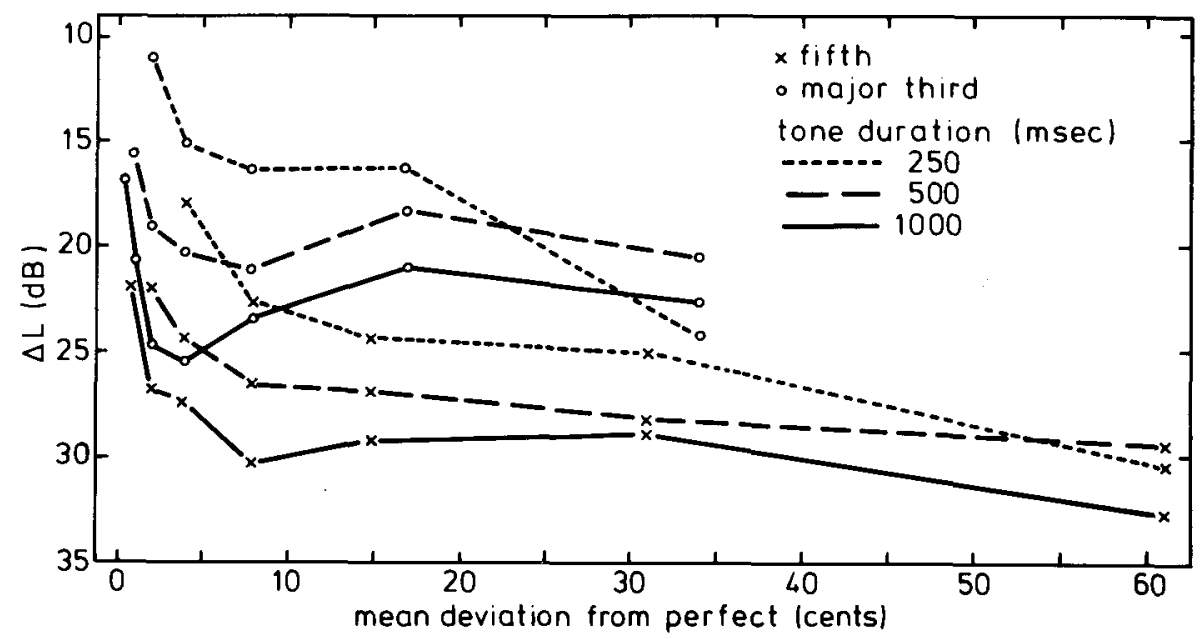

Figure 9. Discrimination thresholds at the different beat frequencies, as given in Figures 6 and 7 , replotted as a function of the corresponding values of mean $|\mathrm{T}|$, with musical interval and tone durntion as parameters. 
would be interesting to test the validity of such a simple rule because, if correct, it would mean that musical intervals, mistuned by only $1 / 10$ th of a cent can be discriminated from a pure interval, provided that its duration is long enough.

Presumably, small fluctuations in the pure interval itself and limitations of human memory will determine the lower bounds for discrimination.

The effect of tone duration becomes smaller and less consistent for the highest beat frequencies. The idea that the effect of beat frequency can be explained by the combined effect of two different mechanisms is supported by the interaction effect of beat frequency and tone duration.

In general, perceptibility of beats is enhanced at longer tone durations. Perception of interval size, however, which is based on the ratio of the fundamental frequencies of the two tones, does not seriously deteriorate as a result of tone duration within the range presented in our experiment (Plomp, Wagenaar, \& Mimpen, 1973).

In sum, both the perception of beats and the perception of interval size may have influenced the DTs. DTs for fifths at beat frequencies higher than $4 \mathrm{~Hz}$ and those for major thirds at beat frequencies higher than $16 \mathrm{~Hz}$ deviate from the U-shaped curve for the detection threshold of beats for simple tones. Assuming a similar sensitivity to beats for simple and complex tones, this fact was tentatively explained by the effect of the perception of interval size. In fact, however, no experimental results on thresholds for the perception of beats for intervals consisting of two complex tones have been reported. Therefore, we designed Experiment 2 to determine the sensitivity to beats for both our mistuned fifths and major thirds. This was done for a large number of beat frequencies, including those presented in the first experiment. Comparison of these beat thresholds (BTs) with the DTs should, it was thought, provide an indirect measure of the contribution to the DTs of the perception of differences in interval size.

\section{EXPERIMENT 2}

\section{Method}

\section{Stimull}

The stimuli were the same as those in Experiment 1.

\section{Apparatus}

The apparatus was identical to that of Experiment 1.

\section{Subjects}

Four musically trained subjects were tested over three sessions, each session on a different day. The subjects had not participated in Experiment 1 and were naive concerning the purposes of the experiment. Three participants were students from the Institute of Musicology at Utrecht, and the fourth was a student at the University of Amsterdam. All subjects were paid for their services.

\section{Experimental Design}

The independent variables were: (1) musical interval (fifth and major third); (2) beat frequency of the first pair of just-noncoinciding harmonics $(.5,1,2,4,6,8,12,16,24$, and $32 \mathrm{~Hz})$; (3) attenuation of tone 1 or tone 2. For every stimulus combination there were four replicas.

\section{Procedure}

On each trial, the subjects were presented with a sequence of mistuned intervals. Each interval had a duration of 1,000 msec. Between the intervals, there was a silent period of $600 \mathrm{msec}$. At the beginning of a trial, $\Delta \mathrm{L}$ was $0 \mathrm{~dB}$. For every successive interval, $\Delta \mathrm{L}$ was increased in steps of $2 \mathrm{~dB}$. During such a descending series, the subjects were instructed to press a key as soon as they no longer perceived any beats. After this response, $\Delta \mathrm{L}$ was decreased in equal steps of $2 \mathrm{~dB}$. In such an ascending series, subjects had to press a second key as soon as they perceived beats again. Within a trial, there were four descending and four ascending series. At the end of a descending series, $\Delta \mathrm{L}$ was increased by a random amount of 2-10 dB. At the end of an ascending series, $\Delta L$ was decreased by a similar variable amount. At the end of every trial, BT was computed by taking the mean of the eight values of $\Delta \mathrm{L}$ at the times that subjects had pressed the keys. A trial was repeated immediately, if the standard deviation of the eight values of $\Delta \mathrm{L}$ was greater than $6 \mathrm{~dB}$. Within a run, both the musical interval and the tone with the variable level were held constant. The presentation order of the different beat frequencies was randomized. Presentation orders of the different runs were balanced according to Latin squares. Each session started with some training series. At the beginning of a run, new waveforms of tones 1 and 2 were determined. The fundamental frequency $f_{1}$ was held constant at $400 \mathrm{~Hz}$, whereas $f_{2}$ depended on interval and beat frequency (see Equation 15). The mistuned intervals were always stretched.

\section{Results}

Again, BTs are presented in terms of level difference $\Delta \mathrm{L}$. BTs for the fifths are given in Figure 10a as a function of beat frequency, with attenuation of tone $2\left(\mathrm{~L}_{1}>\mathrm{L}_{2}\right)$ and tone $1\left(\mathrm{~L}_{1}<\mathrm{L}_{2}\right)$, respectively. In Figure 10b, BTs for the major thirds are given in a similar way. In both figures, the corresponding DTs from Experiment 1 are given as a reference. BTs were subjected to an ANOVA [4 (subjects) $\times 2$ (fifth and major third) $\times 2$ (attenuation of tone 1 or tone 2$) \times 10$ (beat frequencies), all repeated measures]. The effect of subjects, tested against within-cell variance was significant $[F(3,480)=37.8, p<.00001]$; BTs for fifths and major thirds were not significantly different $(p>.72)$; attenuation of tone 1 resulted in lower thresholds than did attenuation of tone $2[F(1,3)=$ 43.5, $\mathrm{p}<.006]$; and BTs were affected by beat frequency $[F(9,27)=7.19, \mathrm{p}<.001]$. A Newman-Keuls paired-comparison test revealed that, in general, thresholds for beat frequencies of $.5,1$, and $2 \mathrm{~Hz}$ are higher than thresholds for 12 and $16 \mathrm{~Hz}(\alpha=.05)$. In view of the specific purposes of this experiment, however, it is more interesting to relate the BTs to the DTs from Experiment 1.

In a comparison of thresholds from Experiments 1 and 2, it was found that the effect of beat frequency for the major thirds was about the same for both BTs and DTs (see Figure 10b). For the fifths, the DTs 

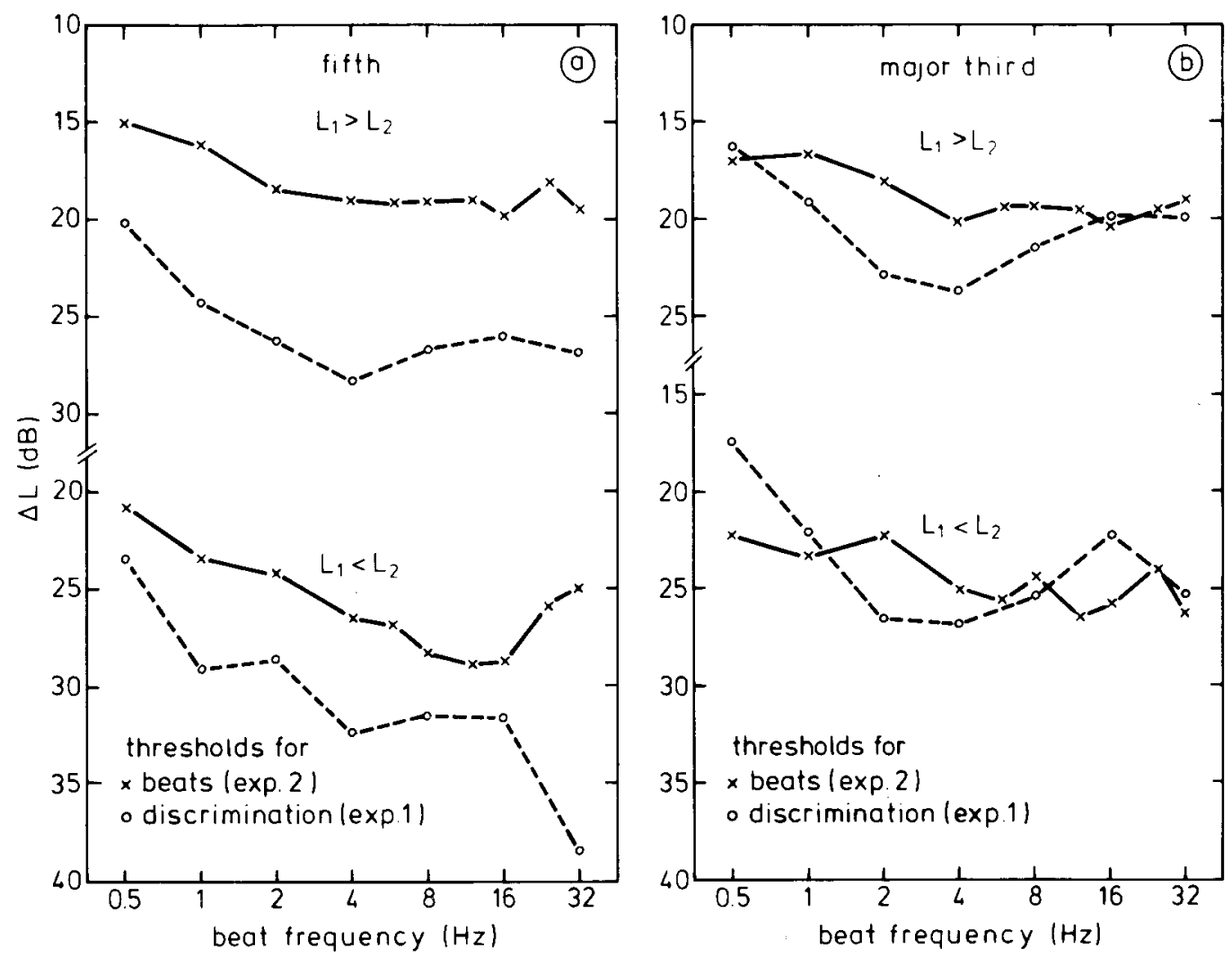

Figure 10. (a) Beat thresholds from Experiment 2 and discrimination thresholds from Experiment 1 for the fifths, plotted as a function of beat frequency with attenuation of tone $2\left(L_{1}>L_{2}\right)$ and attenuation of tone $1\left(L_{1}<L_{2}\right)$, respectlvely. (b) Beat thresholds from Experiment 2 and discrimination thresholds from Experiment 1 for the major thirds, plotted as a function of beat frequency with attenuation of tone $2\left(L_{1}>L_{2}\right)$ and attenuation of tone $1\left(L_{1}<\right.$ $L_{2}$ ), respectively.

were lower than the BTs (see Figure 10a). In the $\mathrm{L}_{1}>\mathrm{L}_{2}$ conditions, the dependence on beat frequency was similar for both types of thresholds. In the $\mathrm{L}_{1}<\mathrm{L}_{2}$ conditions, however, the patterns deviated for beat frequencies higher than $16 \mathrm{~Hz}$. To test the significance of this deviation, these thresholds were subjected to an ANOVA with threshold type as a between-subjects variable and beat frequency as a within-subjects variable. Only the levels of beat frequency that were presented in both experiments were tested. The interaction between threshold type and beat frequency was significant $[F(6,36)=3.81, p<$ $.005]$

\section{Discussion}

The main purpose of Experiment 2 was to ascertain to what extent DTs were due to beats rather than to the perception of differences in interval size. If the perception of differences in interval size contributes to the DTs, DTs and BTs should interact with beat frequency. Deviations between DTs and BTs are expected only for the higher beat frequencies, since the differences in interval width for low beat frequencies are too small to be detected. For the fifth, the inter- action pattern in the $\mathrm{L}_{1}<\mathrm{L}_{2}$ condition strongly suggests that the perception of interval width contributes to DT. For a beat frequency of $32 \mathrm{~Hz}$, DT decreases, whereas BT increases. These interaction effects were not found for either the fifths in the $L_{1}>L_{2}$ conditions or the major thirds in the $\mathrm{L}_{1}>\mathrm{L}_{2}$ and $\mathrm{L}_{1}<\mathrm{L}_{2}$ conditions. Moreover, although the BTs in the latter conditions were higher for the lowest beat frequencies, sensitivity to beats in complex tone intervals does not have a clear maximum such as the one found with superimposed sinusoids.

In Experiment 1, a mean deviation of 34 cents from the pure major third is apparently not large enough for the second mechanism to contribute to the DT. On the other hand, one may ask why the DT did not decrease for the fifth in the $\mathrm{L}_{1}>\mathrm{L}_{2}$ condition. For judgments of interval width to be possible, the levels of both tone 1 and tone 2 should be above masking threshold. It seems plausible to attribute the discrepancy between the $L_{1}<L_{2}$ and $L_{1}>L_{2}$ conditions to the fact that tone 1 is a more effective masker of tone 2 than vice versa.

We had not expected to find that BTs for the fifths would be consistently higher than the DTs, whereas such differences could not be found for the major 
thirds. We can only speculate about the reason. It is possible that the subjects in Experiment 2 were rather conservative in their decisions whether they perceived beats or not. If this was not the case, the data suggest that apart from beats, mistuned intervals near threshold do bear more information distinguishing them from pure intervals than do beats alone. One additional source of information could have been small differences in timbre. The fact that DTs for major thirds were not consistently lower than their corresponding BTs may have been due to differences between the experimental procedures by means of which thresholds were determined. Recall that DTs were investigated by means of the method of constant stimuli, in which fifths and major thirds were randomized within blocks, whereas BTs were determined by employing an adaptive Békésy up-down method. It is possible that the differences between DTs for fifths and major thirds should not be attributed only to level differences between the justnoncoinciding harmonics and to differences in relative interval size by which pure intervals were mistuned, as was done above. The fact that pure major thirds sound rather rough, compared with the smoothsounding pure fifths, may have caused more confusion in discrimination between the pure and mistuned major thirds than would have been the case if interval type had been held constant within blocks.

\section{EXPERIMENT 3}

Three important questions remain unanswered: (1) Are the differences between BTs and DTs due to differences between the employed experimental procedures? (2) Can these differences be attributed to the fact that DTs and BTs were determined for different groups of subjects? (3) What do thresholds for the identification of stretched and compressed intervals look like?

In order to obtain more direct evidence for the relevance of both the perception of beats and the perception of interval width, we designed a new experiment. In Experiment 3, not only DTs and BTs, but also thresholds for identification of the direction of mistuning (ITs) were determined for the same group of subjects in similar experimental conditions.

\section{Method}

\section{Stimull}

The stimuli were the same as those in Experiment 1. The overall level of tones 1 and 2, however, was $80 \mathrm{~dB}$ SPL. Rise and decay times of the tone bursts were 30 and $20 \mathrm{msec}$, respectively. In contrast to Experiment 1, attenuation of either the low tone or the high tone started from the base condition, as depicted in the lefthand panels of Figures $4 \mathrm{a}$ and $4 \mathrm{~b}$. During the experimental runs, the levels of both tone 1 and tone 2 were corrected (see Equation 16) to remain at the same overall level of $80 \mathrm{~dB}$ SPL.

\section{Apparatus.}

The apparatus was identical to that in Experiment 1.
Subjects

Six music students from the Conservatory of Utrecht were tested over six sessions, each session on a different day. All of them had high marks for ear training. The subjects had not participated in Experiment 1 or Experiment 2. They were paid for their services.

\section{Experimental Design}

Thresholds for the identification of the direction of mistuning. The variables were: (1) musical interval (fifth and major third); (2) level difference $\Delta \mathrm{L}$ between the tones ( 0 and $20 \mathrm{~dB}$ ); (3) deviation of the mistuned intervals from perfect intervals $( \pm 2.5, \pm 5$, $\pm 7.5, \pm 10, \pm 15, \pm 20, \pm 25, \pm 30, \pm 35, \pm 40, \pm 45, \pm 50, \pm 60$, \pm 70 , and \pm 80 cents); and (4) attenuation of tone 1 or tone 2 . Variables 1, 2, and 4 resulted in six combinations, because attenuation of tone 1 or tone 2 is relevant only in the $\Delta \mathrm{L}=20 \mathrm{~dB}$ condition. For each of these conditions, the 30 different amounts of mistuning were presented 20 times.

At the end of the experiment it turned out that DTs were lower than $20 \mathrm{~dB}$ in most conditions. Since this experiment was designed mainly to relate the three different thresholds to each other, we decided to determine additional ITs in the following conditions: (1) fifth, $L_{1}<L_{2}, \Delta L=33 \mathrm{~dB}$, (2) fifth, $L_{1}>L_{2}, \Delta L=25 \mathrm{~dB}$, and (3) major third, $\mathrm{L}_{1}<\mathrm{L}_{2}, \Delta \mathrm{L}=25 \mathrm{~dB}$. In these conditions, deviations of the mistuned intervals from perfect were $\pm 10, \pm 20, \pm 30$, $\pm 40, \pm 50, \pm 60, \pm 70$, and \pm 80 cents. Again, the 16 different degrees of mistuning were presented 20 times.

Beat thresholds and discrimination thresholds. The independent variables were: (1) musical interval (fifth and major third), (2) beat frequency of the first pair of just-noncoinciding harmonics $(1,5,15$, and $25 \mathrm{~Hz}$ ), and (3) attenuation of tone 1 or tone 2. For all stimulus combinations, thresholds were determined twice. The dependent variable was $\Delta L$.

\section{Determination of Thresholds for Identification of the Directlon of mistuning}

The percentage of compressed responses $(n=20)$ for the 30 (or 16) different values of mistuning were converted to $z$ scores. The parameters $a$ and $b$ of the linear regression function $z=b+a x$ were determined, where $x$ equals the deviation of the mistuned interval from perfect in cents. From the linear function, thresholds meeting any desired criterion can be derived $(z / a)$. Moreover, the point of subjective purity, $x=-b / a$, that is, the amount of mistuning that results in $50 \%$ stretched and $50 \%$ compressed responses, can be computed as well.

\section{Procedure}

General. The order in which thresholds for the different aspects were determined was balanced: one $(3 \times 3)$ Latin square for the first determination of BTs and DTs and for presentation of half of the trials in the identification task, another $(3 \times 3)$ Latin square for the second determination and the remaining trials in the identification task. Two subjects were assigned to each different presentation order. The duration of the tone bursts was $1,000 \mathrm{msec}$.

Identification thresholds for the direction of mistuning. Each trial consisted of a mistuned interval, which was presented twice. The silent interval between these identical stimuli was $1.3 \mathrm{sec}$. By means of a response box, the subject indicated whether the interval was compressed or stretched, relative to the pure interval. It should be noted (1) that the pure interval was presented only once, at the very beginning of a series of 300 trials, and (2) that no feedback was given. If, at each trial, both the pure and the mistuned interval had been presented, perception of pitch shifts rather than interval width would have been studied. In this experiment, subjects had to imagine a pure interval themselves. Information about the size of this subjective reference interval would have been lost if feedback had been given. Thresholds determined in the conditions described above can be conveniently compared with the DTs, since in the discrimination conditions, the reference was not explicitly given either, but had to be identified by the subjects themselves.

The next trial was presented $1.7 \mathrm{sec}$ after the response to the preceding trial. Within a series, the stimuli were presented in a 
random order. (In a pilot experiment, a double staircase method had been employed: two intermingled series, one for stretched and one for compressed intervals. Since we felt that in this paradigm undesirable cues could be used, the method of constant stimuli was chosen in our main experiment.) Presentation order of the different series, in which both the level of $\Delta \mathrm{L}$ and the musical interval were held constant, was randomized. For values of $\Delta \mathrm{L}>$ $20 \mathrm{~dB}$, the number of trials within a series was reduced to 160 . These series were presented to the same group of subjects in a random order after the main plot, as described in the general procedure, had been finished. Fundamental frequencies were varied from trial to trial in such a way that the central frequency of the mistuned interval was equal to $370 \mathrm{~Hz} \pm 25, \pm 75, \pm 125, \pm 175$, or \pm 225 cents, in a random order. The $f_{1}$ of the interval equaled

$$
f_{1}=f_{c} / 2^{(W+T) / 2,400},
$$

in which $W$ equals $1,200 \log _{2}(q / p)$, and $T$ equals the amount of mistuning relative to $W$. In the same vein, $f_{2}$ was calculated by

$$
f_{2}=f_{c} \cdot 2^{(W+T) / 2,400} \text {. }
$$

A session comprised five or six series. The subjects were not trained.

Beat thresholds. The procedure was similar to that in Experiment 2. The silent period between the intervals, however, was $1 \mathrm{sec}$. The fundamental frequency $f_{1}$ was computed by means of Equation 13, with $f_{c}$ always at $370 \mathrm{~Hz}$. The fundamental frequency $\mathrm{f}_{2}$ was computed by means of Equation 15 . The mistuned intervals were always stretched.

Discrimination thresholds. The task for the subject was to discriminate between pure and mistuned musical intervals in a 2-AFC paradigm. The mistuned interval was presented first in half of the trials and second in the other half of the trials. Between the intervals was a silent period of $1.5 \mathrm{sec}$. By means of a response box, the subject indicated whether the first or the second interval was mistuned. Feedback was given by a red light above the correct button. One subject was visually handicapped; after each correct response, she received feedback by means of a soft tone with a fundamental frequency of $784 \mathrm{~Hz}$ and a duration of $250 \mathrm{msec}$. The next trial was presented $1.7 \mathrm{sec}$ after the response to the preceding trial. DTs were determined by means of an adaptive procedure, in which $\Delta \mathrm{L}$ was increased by $2 \mathrm{~dB}$ after three correct responses and decreased by the same amount after an incorrect response. Each reversal of the direction of change in $\Delta \mathrm{L}$ over successive trials was counted as a turnaround. There were six turnarounds. In the first run, however, $\Delta \mathrm{L}$, being $0 \mathrm{~dB}$ at the very first trial, was increased by $3 \mathrm{~dB}$ after each correct response. The effective DT was taken as the mean value of $\Delta \mathrm{L}$ on the trials that resulted in the last four turnarounds. This value estimates the value of $\Delta \mathrm{L}$ required to produce $\mathbf{7 9 . 4 \%}$ correct responses in a nonadaptive 2-AFC procedure (Levitt, 1971). A series comprised four blocks, one for each beat frequency. Presentation order of the different blocks was randomized. Presentation order of musical interval and attenuation of tone 1 or tone 2, which were held constant within a series, was balanced. At the beginning of a series, new waveforms of tones 1 and 2 , with different $\phi_{n}$ values, were determined. Fundamental frequencies of the pure and mistuned intervals were computed in the same way as in Experiment 1 . The central frequency, $f_{c}$, of the pure interval, however, was equal to $370 \mathrm{~Hz} \pm 25, \pm 75, \pm 125$, \pm 175 , or \pm 225 cents, in a random order. The direction of mistuning (signs in Equations 14 and 15) was randomized. Subjects received two short training series in the first session.

\section{Results and Discussion}

\section{Identification}

For each subject, thresholds at which the direction of mistuning was consistently identified in $75 \%$ of the cases (.67/a) were determined for nine different conditions. For the same conditions, points of subjective purity were also derived. It turned out that the data from one particular subject were very different from those of all the other five subjects. This subject, having a mean IT of 11 cents and a mean point of subjective purity of -35 cents, was therefore dropped from further analysis.

For the remaining five subjects, mean thresholds and mean points of subjective purity are plotted in panels a and b of Figure 11, for each condition separately. The standard deviation, $\sigma_{n-1}$, around these mean values is indicated by bars. Mean ITs for the fifth and the major third are not significantly different and range from about 20 to 30 cents. These findings are more in line with adjustment thresholds for musical intervals than with ITs, as found by Stumpf and Meyer. It is plausible to attribute the discrepancy between Stumpf and Meyer's data and our results to differences in stimulus complexity. For both the fifth and the major third, $t$ tests revealed that a $20-\mathrm{dB}$ attenuation of the higher tone $\left(\mathrm{L}_{1}>\mathrm{L}_{2}\right)$ resulted in significantly higher thresholds than a $20-\mathrm{dB}$ attenuation of the lower tone $\left(L_{1}<L_{2} ; p<.05\right)$. Moreover, for the fifth, the 25-dB attenuation in the $L_{1}>L_{2}$ condition yielded a higher threshold than the 20- and 33-dB attenuation in the $L_{1}<L_{2}$ condition ( $p<.05$ ). These results may be explained by masking effects.

For both the fifth and the major third, and for all different values of $\Delta \mathrm{L}$, the mean points of subjective purity are very close to zero. In other words, mean subjective purity is not significantly different from physical purity.

\section{Discrimination}

DTs were subjected to an ANOVA [5 (subjects) $\times 2$ (fifth or major third) $\times 2\left(\mathrm{~L}_{1}<\mathrm{L}_{2}\right.$ or $\left.\mathrm{L}_{1}>\mathrm{L}_{2}\right) \times 4$ (beat frequency), all repeated measures]. The effect of subjects, tested against within-cell variance, is significant $[F(4,80)=4.8, p<.005]$. DTs for the fifth are about $6 \mathrm{~dB}$ lower than for the major third $[\mathrm{F}(1,4)$ $=137.0, \mathrm{p}<.001]$. Attenuation of tone 1 results in lower DTs than attenuation of tone $2[F(1,4)=38.2$, $\mathrm{p}<.005$ ]. This effect, however, is most prominent for the fifth $[F(1,4)=27.5, p<.01]$. DTs are highest for the lowest beat frequency $[F(3,12)=4.9, p<$ $.02]$. There is a tendency for the effect of beat frequency to depend both on the kind of musical interval $[\mathrm{F}(3,12)=3.3, \mathrm{p}<.05]$ and on the $\mathrm{L}_{1}>\mathrm{L}_{2}$ or $\mathrm{L}_{1}<\mathrm{L}_{2}$ conditions $[\mathrm{F}(3,12)=3.1, \mathrm{p}<.06]$. The interaction patterns can be inspected in Figure 12, in which DTs for the fifth and major third are plotted as a function of the mean deviation from perfect, with attenuation of tone $2\left(\mathrm{~L}_{1}>\mathrm{L}_{2}\right)$ and attenuation of tone $1\left[\mathrm{~L}_{1}<\right.$ $L_{2}$ ), respectively. With respect to the effect of beat frequency for the major third, tests for trend (Winer, $1970)$ revealed that only the cubic trend is significant $[F(1,12)=11.5, p<.01]$. 


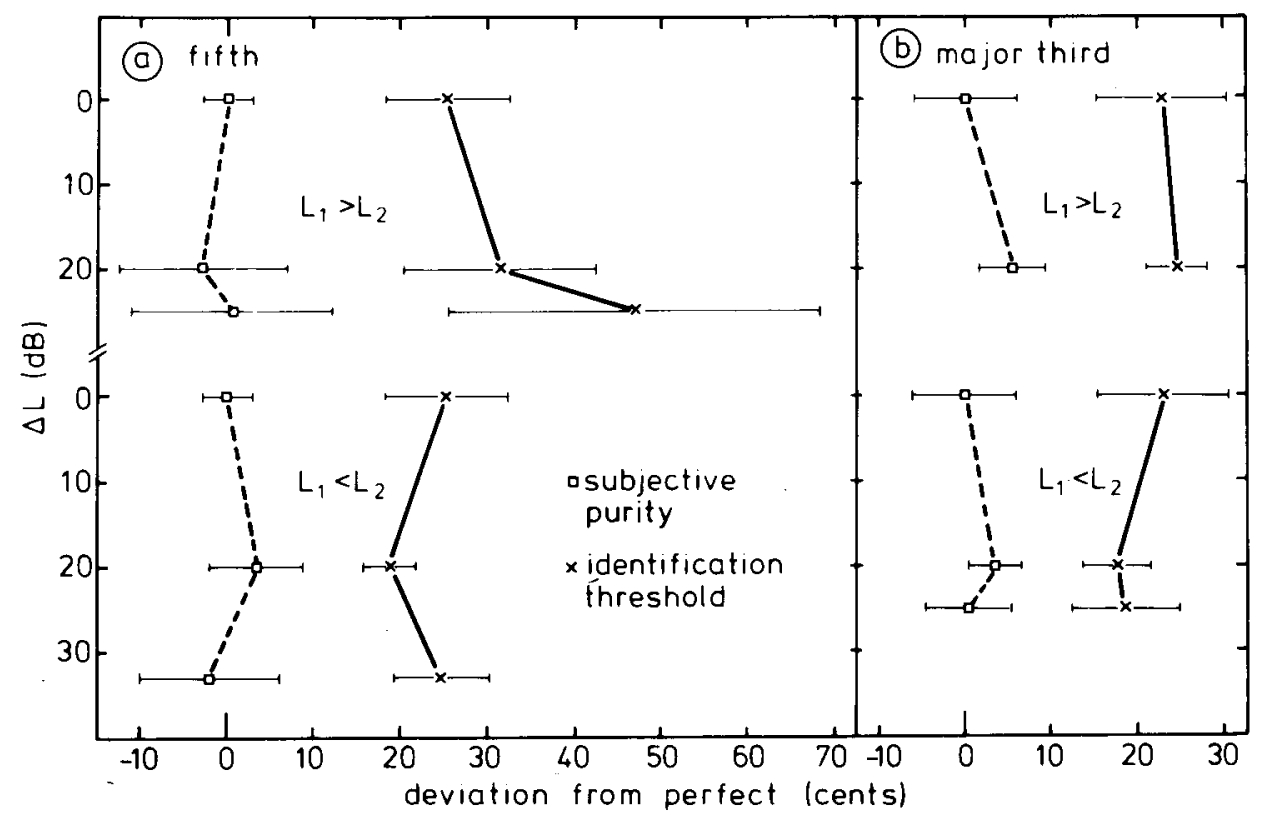

Figure 11. Mean identification thresholds and mean points of subjective purity, plotted with the deviation from a perfect fifth in panel and a pure major third in panel $b$ as the dependent variable. Data are given for the $L_{1}<L_{2}$ and $L_{1}>L_{2}$ conditions and different values of $\Delta L$, separately. Standard deviations, $\sigma_{n-1}$, are Indicated by bars. For every subject, the Identification threshold, as presented here, is based on two parts: (1) a small Interval, added to the pure interval, required for $75 \%$ stretched responses, and (2) a small interval, subtrncted from the pure interval, required for $75 \%$ compressed responses. The identification threshold is equal to the mean of these two tempering intervals. The sign of the mean point of subjective purity Indicates which interval, the first or the second, was larger.

\section{Beats}

BTs are plotted in Figure 12 in the same way as the DTs. An ANOVA with a design similar to that used for the DTs, was carried out. There are substantial differences between subjects $[F(4,80)=31.3$, p < $.00001]$. There is a weak tendency for the thresholds for the fifth to be lower than those for the major third $[F(1,4)=5.6, p<.08]$; the main effect of beat frequency is far from significant $(p>.22)$. Sensitivity to beats is higher in the $\mathrm{L}_{1}<\mathrm{L}_{2}$ condition than in the $L_{1}>L_{2}$ condition $[F(1,4)=10.5, p<.03]$. Combined for the fifth and major third, sensitivity to beats decreases at the highest beat frequency only in the $\mathrm{L}_{1}<\mathrm{L}_{2}$ condition $[\mathrm{F}(3,12)=4.7, \mathrm{p}<.02]$.

\section{Comparison of Discrimination and Beat Thresholds}

DTs and BTs were subjected to an ANOVA [5 (subjects) $\times 2$ (DT or BT) $\times 2$ (fifth or major third) $\times 2\left(\mathrm{~L}_{1}<\mathrm{L}_{2}\right.$ or $\left.\mathrm{L}_{1}>\mathrm{L}_{2}\right) \times 4$ (beat frequency), all repeated measures]. BTs are significantly higher than DTs only for the fifth $[F(1,4)=17.5, p<.01]$. Combined for the fifth and major third, the differences between the DT and BT increase at the highest beat frequency only in the $\mathrm{L}_{1}<\mathrm{L}_{2}$ condition $[\mathrm{F}(3,12)=$ $4.5, \mathrm{p}<.02]$. The patterns of DTs and BTs, as pre- sented in Figure 12, can be compared with the corresponding patterns from Figure 10. In fact, the effects for the fifth in the $L_{1}>L_{2}$ and $L_{1}<L_{2}$ conditions, and for the major third in the $\mathrm{L}_{1}>\mathrm{L}_{2}$ condition, were confirmed. For the major third in the $\mathrm{L}_{1}<\mathrm{L}_{2}$ condition, however, the significant deviation of DT and BT at the greatest mistuning $(t=2.33$, $p<.05)$ suggests that the perception of interval width, which was claimed to play a part at the high beat frequencies in the $\mathrm{L}_{1}<\mathrm{L}_{2}$ condition of the fifth (see Figure 10a), has contributed to DT as well.

One may wonder how much weight should be attributed to the fact that the mean BTs are higher than the corresponding DTs. The criterion on which decisions for the presence or absence of beats are based had to be chosen by our subjects themselves. The fact that $34.5 \%$ of the variance in BTs from Experiment 3 was explained by the main effect of subjects, whereas this percentage was only 4.7 for the DT shows that this criterion was strongly dependent on the subject. Moreover, correlation coefficients between DTs and BTs per se are low. On the other hand, during the experiment, the criteria for every subject remained relatively stable, since within-cell variance for BTs and DTs were about the same: $36 \%$ and $40 \%$, respectively. Therefore, it is more appropriate to com- 


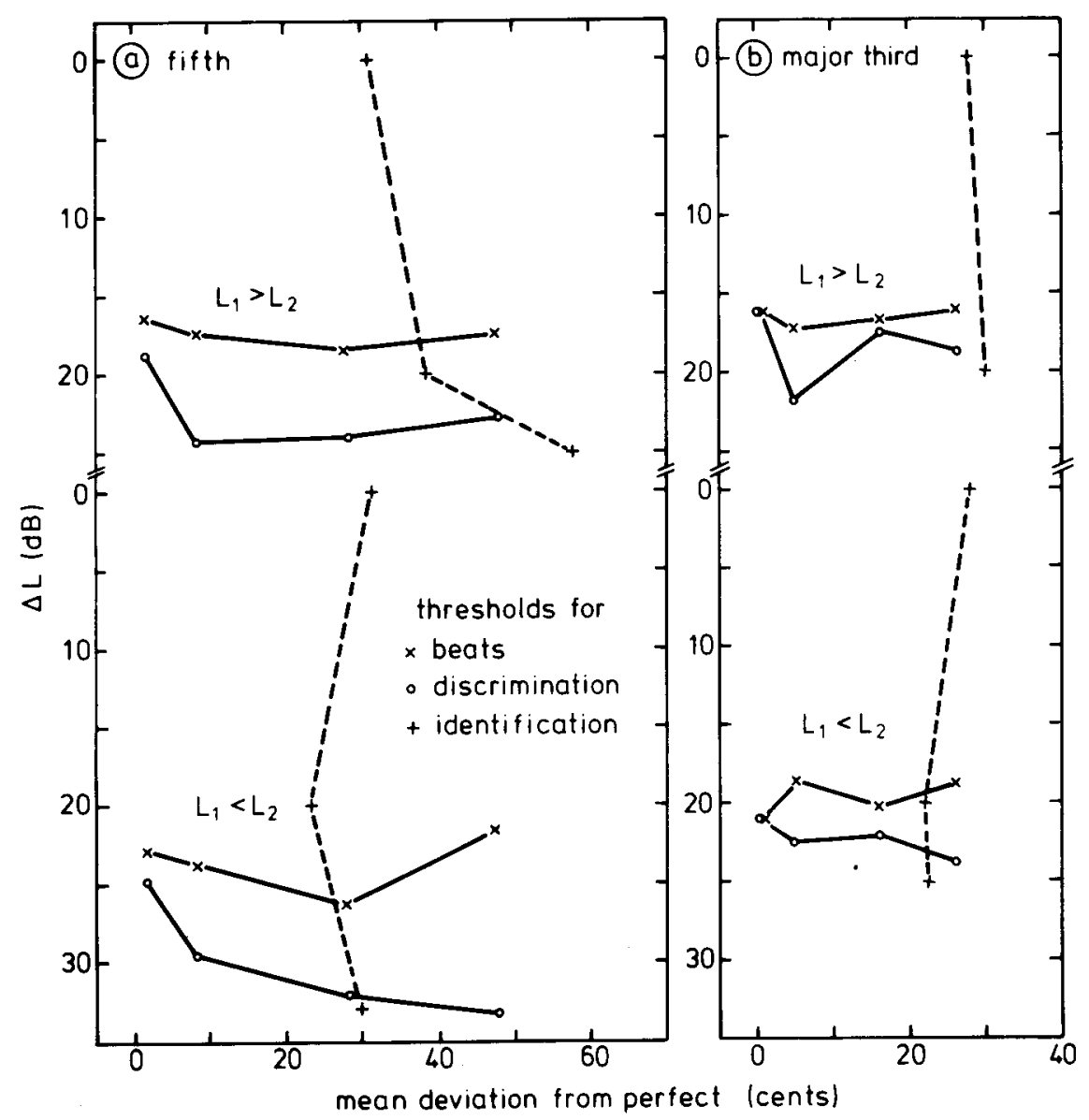

Figure 12. Beat thresholds and discrimination thresholds, plotted as a function of the mean deviation from perfect with attenuation of tone $2\left(L_{1}>L_{2}\right)$ and attenuation of tone $1\left(L_{1}<\right.$ $L_{2}$ ), respectively. Also presented are identification thresholds from Figure 11, but now for a performance criterion of $79.4 \%$, with the deviation from perfect as the dependent. Thresholds are given for the $L_{1}>L_{2}$ and $L_{1}<L_{2}$ conditions and for the different $\Delta L$ values, separately. The different thresholds for the fifth and the major third are given in panels a and $b$, respectively.

pare the patterns of the thresholds and, as we shall see below, define changes in threshold levels relative to thresholds from comparable conditions.

\section{Discrimination, Beat, and Identification \\ Thresholds Together}

For the data from Experiment 3, the plausibility of the idea that both sensitivity to beats and the perception of interval width may have contributed to the DT can be supported more directly because ITs are available. Therefore, ITs from Figure 11, but now, like the DTs, for a performance criterion of $79.4 \%$, are replotted in Figure 12. The specific interaction patterns between BTs, ITs, and DTs can be tentatively described by the following rule. The DT depends on BT and IT in a compensatory manner. For example, if the BT at the highest beat frequency is relatively high, but the IT is low, the DT remains low (fifth and major third, $\mathrm{L}_{1}<\mathrm{L}_{2}$ ). If the IT is high, but the BT is relatively low, the DT remains low (fifth and major third, $L_{1}>L_{2}$ ). A consequence of this rule is that if both the BT and the IT are high, the DT has to be high as well. We realize that the patterns shown in Figure 12 are no more than suggestive. A stronger relation between DT and IT can be demonstrated if we correlate the individual decrements of the DT for a beat frequency of $25 \mathrm{~Hz}$, relative to the mean of the corresponding DTs for beat frequencies of 1,5 , and $15 \mathrm{~Hz}$, with the individual ITs for the corresponding $\Delta \mathrm{L}=20 \mathrm{db}$ conditions. The correlation coefficients are given in the first column of Table 1. Especially for the fifth, where the mean amount of mistuning at a beat frequency of $25 \mathrm{~Hz}$ is 22 cents higher than it is for the major third, the $r$ values of -.83 and -.73 are high. If we correlate the relative decrease of the DT with a similar decrease of the sensitivity to beats, high correlations are found for the $L_{1}>L_{2}$ conditions (see second column of 
Table 1

Prediction of the Relative Decrease of the Discrimination Threshold $\left(X_{1}\right)$ From Identification Threshold $\left(X_{2}\right)$ and Relative Decrease of the Beat Threshold $\left(X_{3}\right)$ by Means of the Multiple Linear Regression Equation $X_{1}^{\prime}=m_{1} X_{2}+m_{2} X_{3}$

\begin{tabular}{llccccc}
\hline \multicolumn{1}{l}{ Condition } & & $\mathrm{r}_{12}$ & $\mathrm{r}_{13}$ & $\mathrm{r}_{1.23}$ & $\mathrm{~m}_{1}$ & $\mathrm{~m}_{2}$ \\
\hline \multirow{2}{*}{ Fifth } & $\mathrm{L}_{1}<\mathrm{L}_{2}$ & -.83 & .25 & .93 & -.91 & .42 \\
& $\mathrm{~L}_{1}>\mathrm{L}_{2}$ & -.73 & .86 & .94 & -.42 & .67 \\
\multirow{2}{*}{ Major Third } & $\mathrm{L}_{1}<\mathrm{L}_{2}$ & -.19 & .37 & .45 & .51 & .81 \\
& $\mathrm{~L}_{1}>\mathrm{L}_{2}$ & -.56 & .85 & .85 & -.11 & .79 \\
\hline
\end{tabular}

Note-All scores were normalized. For both the discrimination and beat thresholds, the relative decrease is defined as the difference between the thresholds at a beat frequency of $25 \mathrm{~Hz}$ and the mean of the corresponding thresholds at beat frequencies of 1,5 , and $15 \mathrm{~Hz}$. For all conditions, identification thresholds were taken from the corresponding $\Delta L=20 \mathrm{~dB}$ conditions. The different correlation coefficients and regression weights are given separately for the fifths and major thirds in both the $L_{1}<L_{2}$ and $L_{1}>L_{2}$ conditions.

Table 1). As shown in the third column in Table 1, the relative decrease of the DT can be predicted even better, if both the IT and the relative decrease of the BT are considered simultaneously. For each condition, the relative contribution of the perception of interval size and sensitivity to beats to the decrease of the DT at the highest beat frequency can be determined. For the fifth in the $\mathrm{L}_{1}<\mathrm{L}_{2}$ condition, the explained variance increases by $17 \%$ if sensitivity to beats is also taken into account. In the $\mathbf{L}_{1}>\mathbf{L}_{2}$ conditions, however, the explained variance increases by $35 \%$ for the fifth and by $41 \%$ for the major third. Only for the major third in the $\mathrm{L}_{1}<\mathrm{L}_{2}$ condition is neither the IT nor the increased sensitivity to beats a very effective predictor of the relative decrease of the DT. For the remaining conditions, however, the compensatory character, in which the DT depends on the IT and BT, is suggested by the values of the regression weights, which are given in columns 4 and 5 of Table 1. For each condition, prediction is optimized by giving either identification $\left(m_{1}\right)$ or beats $\left(m_{2}\right)$ the highest weight.

\section{GENERAL CONCLUSIONS}

Combining the results from Experiments 1 and 3, we may conclude that DTs (1) are higher for major thirds than for fifths, (2) are highest for the lowest beat frequencies, and (3) decrease with increasing tone duration. In addition, (4) the effect of tone duration is highest at low beat frequencies.

From Experiment 3, we may conclude that (5) ITs are about the same for fifths and major thirds, deviations from perfect at threshold ranging from 20 to 30 cents, and (6) mean subjective purity is not significantly different from physical purity.
Combining the results from Experiments 2 and 3, we may conclude that (7) sensitivity to beats for mistuned fifths and major thirds is about the same and (8) beat frequency affects sensitivity to beats in only some conditions.

If we are willing to relate the patterns, rather than the absolute values of the different thresholds, we may conclude that (9) discrimination is mainly determined by sensitivity to beats. Detailed analysis, however, indicates that (10) the perception of interval size has been a relevant aspect in discrimination, especially for the fifths in the $L_{1}<L_{2}$ condition.

In the introduction, we proposed that knowledge of our sensitivity to mistuning might provide at least one criterion for the evaluation of different tuning systems. In this context, the first conclusion may be related to the fact that in the tuning system that is applied most frequently, that is, the equal temperament, fifths are mistuned only slightly ( 2 cents), taking the considerable mistuning of the major thirds (14 cents) into the bargain. Moreover, the decreased sensitivity to mistuning of the major third for beat frequencies around, say, $15 \mathrm{~Hz}$, as found in Experiments 1 and 3 , suggests once more how to optimize the purity of the fifth and major third. From conclusions 3 and 4 , it can be predicted that the tolerance of mistuned intervals is higher in passages with short tones than with sustained tones, provided the mistuning is not too large. Evidently, tolerance of mistuned intervals, presented within a musical context, will depend on both the sensitivity to mistuning, as has been determined here for isolated intervals, and the musical context itself. The fifth conclusion squares with the general experience that Pythagorean thirds and, especially, the wolf from regular mean tone temperament are easily identified.

\section{REFERENCE NOTE}

1. Rakowski, A. Tuning of isolated musical intervals. Paper presented at the 91 st Meeting of the Acoustical Society of America, Washington, D.C., 1976.

\section{REFERENCES}

Barbour, J. M. Musical logarithms. Scripta Mathematica, 1940, 7, 21-31.

Burns, E. M., \& W ARD, W. D. Categorical perception-phenomenon or epiphenomenon: Evidence from experiments in the perception of melodic musical intervals. Journal of the Acoustical Society of America, 1978, 63, 456-468.

Delezenne, M. Mémoire sur les valeurs numériques des notes de la gamme. Recueil des travaux de la Société des Sciences de Lille, 1826-1827, 1-71.

Ebel, H. Das Hören von Amplitudenmodulationen. Acustica, 1952, 2, 246-250.

HARRis, J. D. Loudness discrimination. Journal of Speech and Hearing Disorders, 1963, Monograph Supplement II.

Helmholtz, H. L. F. von. [On the sensations of tone as a physiological basis for the theory of music] (A. J. Ellis, trans.). New York: Dover, 1954. (Originally published, 1877.) 
Kameoka, A., \& Kuriyagawa, M. Consonance theory, Part II: Consonance of complex tones and its calculation method. Journal of the Acoustical Society of America, 1969, 45, 1460-1469.

Killam, R. N., Lorton, P. V., \& Schubert, E. D. Interval recognition: Identification of harmonic and melodic intervals. Journal of Music Theory, 1975, 19, 212-235.

KLING, J. W., \& RigGs, L. A. Woodworth and Schlosberg's experimental psychology (3rd ed.). New York: Holt, Rinehart and Winston, 1972.

LEviTT, H. Transformed up-down methods in psychoacoustics. Journal of the Acoustical Society of America, 1971, 49, 467-477.

MaIwald, D. Ein Funktionsschema des Gehörs zur Beschreibung der Erkennbarkeit kleiner Frequenz- und Amplitudenänderungen. Acustica, 1967, 18, 81-92.

Moran, H., \& Pratt, C. C. Variability of judgments on musical intervals. Journal of Experimental Psychology, 1926, 9, 492-500.

PikLER, A. G. Logarithmic frequency systems. Journal of the Acoustical Society of America, 1966, 39, 1102-1110.

Plomp, R. Aspects of tone sensation. A psychophysical study. London: Academic Press, 1976.

Plomp, R., \& Levelt, W. J. M. Tonal consonance and critical bandwidth. Journal of the Acoustical Society of America, 1965, 38, 548-560.

Plomp, R., Wagenaar, W. A., \& Mimpen, A. M. Musical interval recognition with simultaneous tones. Acustica, 1973, 29, 101-109.

RIEsz, R. R. Differential intensity sensitivity of the ear for pure tones. Physical Review, 1928, 31, 867-875.
SснӧNE, P. Messungen zur Schwankungsstärke von amplitudenmodulierten Sinustönen. Acustica, 1979, 41, 252-257.

Siegel, J. A., \& Siegel, W. Absolute identification of notes and intervals by musicians. Perception \& Psychophysics, 1977, 21, 143-152. (a)

Siegel, J. A., \& Siegel, W. Categorical perception of tonal intervals: Musicians can't tell sharp from flat. Perception \& Psychophysics, 1977, 21, 399-407. (b)

STUMPF, C., \& MEYER, M. Maassbestimmungen über die Reinheit consonanter Intervalle. Beiträge zur Akustik und Musikwissenschaft, 1898, 2, 84-167.

TerhardT, E. On the perception of periodic sound fluctuations (roughness). Acustica, 1974, 30, 201-213.

TerhardT, E. Ein psychoakustisch begrundetes Konzept der musikalischen Konsonanz. Acustica, 1976, 36, 121-137.

WINER, B. J. Statistical principles in experimental design (International student ed.). London: McGraw-Hill, 1970.

YounG, R. W. Terminology for logarithmic frequency units. Journal of the Acoustical Society of America, 1939, 11, 134-139.

Zatorre, R. J., \& Halpern, A. R. Identification, discrimination, and selective adaptation of simultaneous musical intervals. Perception \& Psychophysics, 1979, 26, 384-395.

Zwicker, E. Die Grenzẹn der Hörbarkeit der Amplitudenmodulation und der Frequenzmodulation eines Tones. Acustica, $1952,2,125-133$.

(Manuscript received April 27, 1982; revision accepted for publication July 12,1982 .) 